\title{
Nickel catalysts bearing bidentate $\alpha$-aminoaldimines for ethylene polymerization-independent and cooperative structure/reactivity relationship resulting from unsymmetric square planar coordination $\uparrow$
}

\author{
Feng-Zhao Yang, Yi-Chun Chen, Ya-Fan Lin, Kuo-Hsuan Yu, Yi-Hung Liu, Yu Wang, Shiuh-Tzung Liu and \\ Jwu-Ting Chen*
}

Received 19th August 2008, Accepted 30th October 2008

First published as an Advance Article on the web 8th January 2009

DOI: $10.1039 / b 814423 k$

Ethylene polymerization catalyzed by $\mathrm{Ni}$ (II) complexes that bear new bidentate ligands with a functional hybrid of amine and imine has been studied. A class of new $\alpha$-aminoaldimines and their nickel complexes $\left[\mathrm{R}^{1} \mathrm{R}^{2} \mathrm{NCMe}{ }_{2} \mathrm{CH}=\mathrm{N}\left(2,6-\mathrm{R}_{2}^{3} \mathrm{C}_{6} \mathrm{H}_{3}\right)\right] \mathrm{NiBr}_{2}\left(\mathrm{R}^{1}=\mathrm{R}^{2}=\mathrm{Me}, \mathrm{R}^{3}=\mathrm{Me}(\mathrm{Ni}-\mathbf{1 b}) ; \mathrm{R}^{3}={ }^{\mathrm{i}} \mathrm{Pr}\right.$ (Ni-1c); $\mathrm{R}^{1}=\mathrm{R}^{2}=\mathrm{Et}, \mathrm{R}^{3}=\mathrm{H}(\mathrm{Ni}-2 \mathbf{a}) ; \mathrm{Me}(\mathrm{Ni}-2 \mathbf{b}) ;{ }^{i} \operatorname{Pr}(\mathrm{Ni}-2 \mathrm{c}) ; \mathrm{R}^{1}=\mathrm{R}^{2}={ }^{n} \operatorname{Pr}, \mathrm{R}^{3}={ }^{\mathrm{i}} \operatorname{Pr}(\mathrm{Ni}-3 \mathbf{c})$; $\left.\left(\mathrm{R}^{1} \mathrm{R}^{2}\right)=c-\mathrm{C}_{3} \mathrm{H}_{6} \mathrm{R}^{3}={ }^{i} \operatorname{Pr}(\mathrm{Ni}-4 \mathrm{c}) ;\left(\mathrm{R}^{1} \mathrm{R}^{2}\right)=c-\mathrm{C}_{4} \mathrm{H}_{8}, \mathrm{R}^{3}={ }^{i} \operatorname{Pr}(\mathrm{Ni}-5 \mathrm{c})\right)$ were synthesized. The molecular structures of six nickel complexes were determined by X-ray crystallography, showing distorted tetrahedral configurations. The SQUID data of Ni-1c confirms its ground state of triplet spin. Using methylaluminoxanes (MAO) as the activator, the nickel complexes are found to catalyze ethylene polymerization under moderate pressure and ambient temperature. The activity reaches to $10^{6} \mathrm{~g}$ PE mol $\mathrm{Ni}^{-1} \mathrm{~h}^{-1}$, and increases with the ethylene pressure in the range of 14-28 bar. The highly branching PE products have $\mathrm{M}_{n} \sim 10^{5}$ with $\mathrm{PDI}<2$. The amine and imine functionalities demonstrate independent control to the polymerization reactions, wherein the activity appears to be facilitated by using the catalysts installed with bulky imino substituents as well as with less sterically hindered amino substituents. This is ascribed to the $C_{2}$ unsymmetric coordination in the square planar resting state in which the bulky polymer chain prefers cis to the imine and the small ethylene monomer is cis to the amine.

\section{Introduction}

The usage of late transition metal catalysts bearing the designed ligands with constrained geometry for olefin polymerization has acquired tremendous attention. ${ }^{1}$ Such catalysts are expected to not only demonstrate promising activity, but also to confer a characteristic control to the reaction course as well as to the polymer properties, particularly distinguishable from the reactions caused by the known catalysts of early transition metals. ${ }^{2}$ Among the studied systems, the catalysts with diimine ligands with the bulky substituents have been proved to represent a paradigm. It leads to immense research in seeking for the ligand control with steric bulkiness. ${ }^{3}$

In another aspect, the quest for new ligands with the hybrid donating functionalities still remains a rising field since the discovery of the SHOP process. ${ }^{4}$ It is generally believed that the unsymmetric bidentate ligands potentially enable to afford the distinct influence to the metal in the regards of both structure and reactivity control. ${ }^{5,6}$ Some recent studies reveal that ethylene polymerization may be catalyzed by the bidentates with $\mathrm{N}-\mathrm{O}$, $\mathrm{P}-\mathrm{N}$ and $\mathrm{N}-\mathrm{N}$ ' donor combination. ${ }^{7-9}$ Curiously, the bidentates

Department of Chemistry, National Taiwan University, No 1, Section 4, Roosevelt Road, Taipei, Taiwan 106.E-mail: jtchen@ntu.edu.tw; Fax: +886 22363 6359; Tel: +886233661659

$\dagger$ Electronic supplementary information (ESI) available: SQUID data of Ni-1c. CCDC reference numbers 682282 and 699109-699113. For ESI and crystallographic data in CIF or other electronic format see DOI: $10.1039 / \mathrm{b} 814423 \mathrm{k}$ comprising amine and imine are left as a relatively unripe field in catalysis. ${ }^{10}$ We report herein that nickel catalysts bearing new $\alpha$-aminoaldimine ligands are found to be highly active to ethylene polymerization. ${ }^{11}$ In such a complex system, each coordinating functionality displays independent but cooperative structure-to-reactivity relationship. These ligands thus provide the rare examples of different donor functionalities that can convey selective geometrical isomerism, and further affect the reactivity of ethylene polymerization.

\section{Results and discussion}

\section{Synthesis and spectroscopic characterization}

New $\alpha$-aminoaldimines in the form of $\mathrm{R}^{1} \mathrm{R}^{2} \mathrm{NCMe}{ }_{2} \mathrm{CH}=\mathrm{N}(2,6$ $\left.\mathrm{R}^{3}{ }_{2} \mathrm{C}_{6} \mathrm{H}_{3}\right)(\mathrm{L})$ were synthesized via amination of $\alpha$-bromoaldehyde followed by condensation with the aniline derivatives as shown in Scheme 1.12

The substitution reactions of $(\mathrm{DME}) \mathrm{NiBr}_{2}(\mathrm{DME}=1,2-$ dimethoxyethane) with $\mathbf{L}$ generates the neutral complexes $\left[\mathrm{R}^{1} \mathrm{R}^{2} \mathrm{NCMe}{ }_{2} \mathrm{CH}=\mathrm{N}\left(2,6-\mathrm{R}_{2}^{3} \mathrm{C}_{6} \mathrm{H}_{3}\right)\right] \mathrm{NiBr}_{2}\left(\mathrm{R}^{1}=\mathrm{R}^{2}=\mathrm{Me}, \mathrm{R}^{3}=\right.$ $\mathrm{Me}(\mathrm{Ni}-1 \mathbf{b}) ; \mathrm{R}^{3}={ }^{i} \operatorname{Pr}(\mathrm{Ni}-1 \mathbf{c}) ; \mathrm{R}^{1}=\mathrm{R}^{2}=\mathrm{Et}, \mathrm{R}^{3}=\mathrm{H}(\mathrm{Ni}-\mathbf{2 a}) ; \mathrm{Me}$ (Ni-2b); $\operatorname{Pr}(\mathrm{Ni}-2 \mathrm{c}) ; \mathrm{R}^{1}=\mathrm{R}^{2}={ }^{n} \operatorname{Pr}, \mathrm{R}^{3}={ }^{i} \operatorname{Pr}(\mathrm{Ni}-3 \mathrm{c}) ;\left(\mathrm{R}^{1} \mathrm{R}^{2}\right)=$ $\left.c-\mathrm{C}_{3} \mathrm{H}_{6}, \mathrm{R}^{3}={ }^{i} \operatorname{Pr}(\mathrm{Ni}-4 \mathrm{c}) ;\left(\mathrm{R}^{1} \mathrm{R}^{2}\right)=c-\mathrm{C}_{4} \mathrm{H}_{8}, \mathrm{R}^{3}={ }^{i} \operatorname{Pr}(\mathrm{Ni}-5 \mathrm{c})\right)$. The violet dibromonickel complexes are generally soluble in $\mathrm{CH}_{2} \mathrm{Cl}_{2}$ or $\mathrm{CHCl}_{3}$, and appear to be hygroscopic. The SQUID measurement for Ni-1c gives $\mu=3.18 \mathrm{BM}$ at $295 \mathrm{~K}$, indicating a ground state of triplet spin. 


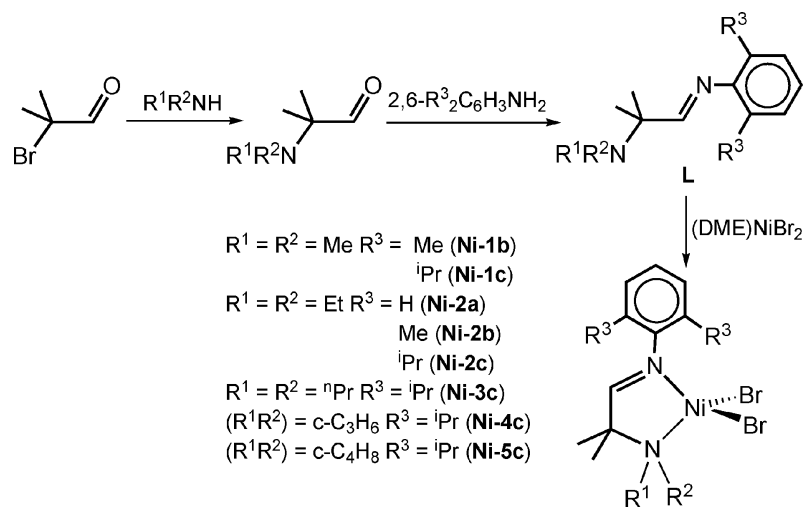

Scheme 1 Synthesis of ligands and nickel catalysts.

\section{X-Ray structural analysis}

The single crystals of the nickel complexes were grown from $\mathrm{CH}_{2} \mathrm{Cl}_{2}-\mathrm{Et}_{2} \mathrm{O}$. The X-ray diffraction data, listed in Table 3, were collected using $3 \mathrm{~kW}$ sealed-tube $\operatorname{MoK} \alpha$ radiation $(\lambda=0.7103 \AA)$. The data were processed using the DENZO method and the structure were solved and refined by the SHELXTL program. ${ }^{13}$ Multi-scan absorption correction was applied, and decay was negligible. All refinements were carried out by full-matrix leastsquares using anisotropic displacement parameters for all nonhydrogen atoms. All the hydrogen atoms were added at calculated positions.

ORTEP drawings of Ni-1b, Ni-1c, Ni-2a, Ni-2b, Ni-4c and Ni$\mathbf{5 c}$ with thermal ellipsoids at 50\% probability are shown in Fig. 1. The four-coordinate molecular structures in distorted tetrahedral geometry are confirmed. Such results explains the paramagnetism shown by Ni-1c. The bond parameters are listed in Table 4 .

The distances of the $\mathrm{Ni}-\mathrm{N} 1\left(\mathrm{sp}^{2}\right)$ bonds are in the range of

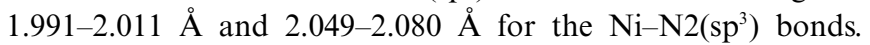
The data are consistent with the nickel-nitrogen bonds with the imine and amine ligands, respectively. ${ }^{14}$ The angles of N1-Ni-N2 are in the range of $81.7-83.3^{\circ}$, which are comparable with those in the diimine complexes. ${ }^{3 c, g, 14 c, d}$ The angles of $\mathrm{Br} 1-\mathrm{Ni}-\mathrm{Br} 2$ are in the larger range of 116.1-123.6. $6^{\circ}$. Complex Ni-2a has the particularly large $\mathrm{Br} 1-\mathrm{Ni}-\mathrm{Br} 2$ angle. The average distances of the $\mathrm{Ni}-\mathrm{Br}$ bonds are in the range of 2.3338-2.3654 $\AA$. The Ni-Br distance of $\mathrm{Ni}-\mathbf{2 a}(2.3536$ and $2.3772 \AA$ ) are also longer than those in other analogues. The $\mathrm{Ni}-\mathrm{N} 1-\mathrm{C} 1$ angles are in the range of $111.5-114.5^{\circ}$ and $103.3-108.3^{\circ}$ for the $\mathrm{Ni}-\mathrm{N} 2-\mathrm{C} 2$ angles. All these structural features give solid support to the authentic amineimine bidentate coordination to the nickel(II) center. The nonplanar five-membered metallacycles imply that the amino and imino substituents will be able to provide quite different steric effect to their $c i s$ coordination sites. ${ }^{12}$

\section{Ethylene polymerization}

The nickel complexes have been found to be active catalysts for ethylene polymerization with the assistance of methylaluminoxanes at ambient temperature. The data are presented in Table 1. At a pressure of $28 \mathrm{bar}$ and $25^{\circ} \mathrm{C}$, the activity of $\mathrm{Ni}-1 \mathrm{~b}$ reaches to $10^{6} \mathrm{~g} \mathrm{~mol} \mathrm{Ni}^{-1} \mathrm{~h}^{-1}$. In general, the PE products are of hyperbranch and have $M_{n}$ of $10^{5}$ with $M_{w} / M_{n}<2$. The $T_{\mathrm{g}}$ of PE are in the

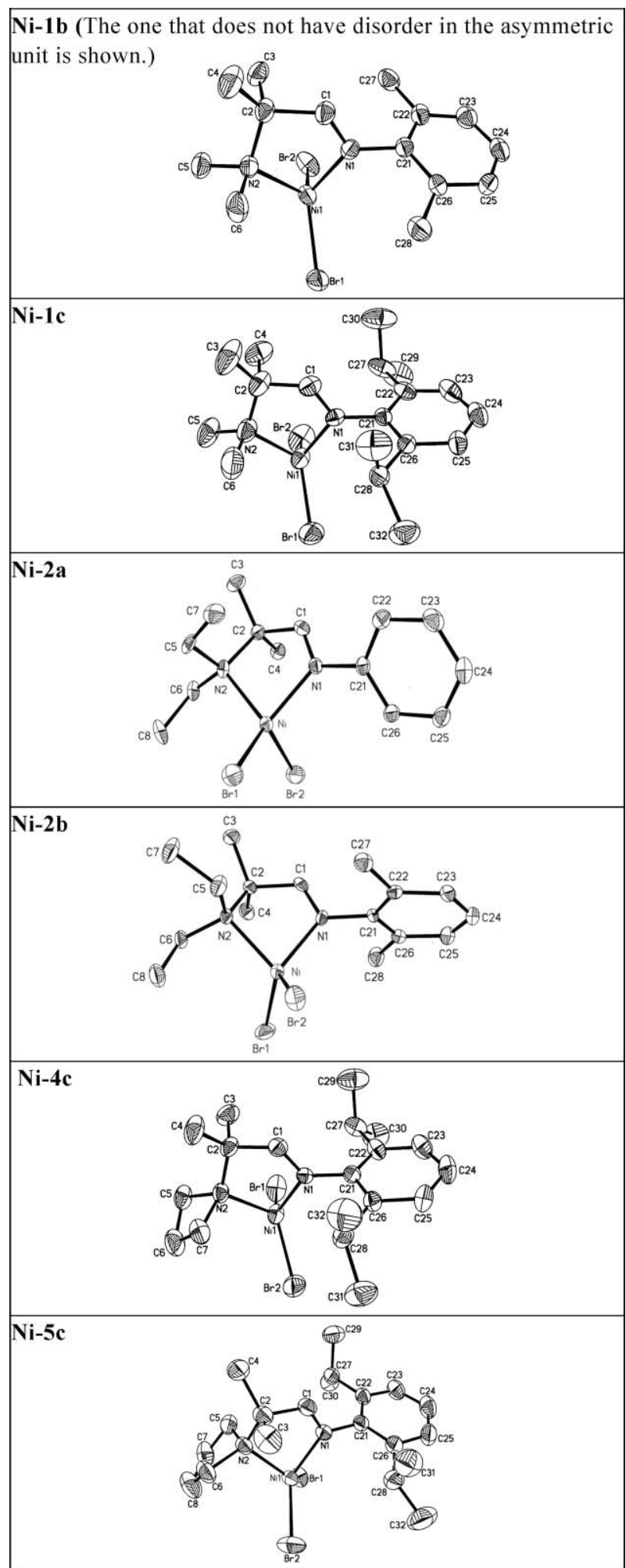

Fig. 1 ORTEP drawings of the nickel complexes. All hydrogen atoms are omitted for clarity. Ni-1b (the one that does not have disorder in the asymmetric unit is shown).

region of -40 to $-50{ }^{\circ} \mathrm{C}$, but no observable $T_{\mathrm{m}}$. TGA data show less than $10 \%$ weight loss until $390^{\circ} \mathrm{C}$.

Entries 1-5 show that the catalysts Ni-1b are active within 10 min. For Ni-2b, as illustrated in the entries 9 and 10, the 
Table 1 Data of ethylene polymerization

\begin{tabular}{|c|c|c|c|c|c|c|c|}
\hline & Catalyst $/ \mu \mathrm{mol}^{a}$ & $\mathrm{C}_{2} \mathrm{H}_{4} /$ bar & $T_{\text {rxn }} / \mathrm{h}$ & $\mathrm{TOF}^{b} \times 10^{-3}$ & $M_{n}^{c} \times 10^{-3}$ & PDI & Branch $/ \times 10^{3} \mathrm{C}^{d}$ \\
\hline 1 & $\mathrm{Ni}-1 \mathbf{b}(41)$ & 14 & $1 / 6$ & 334 & 236 & 1.38 & 62 \\
\hline 2 & Ni-1b (41) & 17 & $1 / 6$ & 516 & 279 & 1.35 & 121 \\
\hline 4 & Ni-1b (41) & 24 & $1 / 6$ & 1030 & 291 & 1.32 & 112 \\
\hline 5 & Ni-1b (41) & 28 & $1 / 6$ & 1106 & 226 & 1.66 & 78 \\
\hline 6 & $\mathrm{Ni}-1 \mathrm{c}(39)$ & 28 & $1 / 6$ & 1085 & 356 & 1.21 & 112 \\
\hline 7 & Ni-1c (43) & 28 & $1 / 2$ & 1186 & 378 & 1.44 & 106 \\
\hline 10 & $\mathrm{Ni}-2 \mathbf{b}(22)$ & 17 & 24 & 25 & 393 & 1.23 & 118 \\
\hline 11 & $\mathrm{Ni}-2 \mathbf{c}(22)$ & 17 & 3 & 52 & 238 & 1.69 & 143 \\
\hline 12 & Ni-3c (22) & 17 & 3 & 74 & 108 & 2.58 & 103 \\
\hline 13 & $\mathrm{Ni}-\mathbf{4 c}(22)$ & 17 & $1 / 3$ & 682 & 297 & 1.25 & 86 \\
\hline 14 & $\mathrm{Ni}-\mathbf{5 c}(22)$ & 17 & $1 / 3$ & 500 & 227 & 1.26 & 79 \\
\hline
\end{tabular}

${ }^{a}$ All runs were carried out in $100 \mathrm{~mL}$ toluene at $25{ }^{\circ} \mathrm{C},[\mathrm{Al}] /[\mathrm{Ni}]=480 .{ }^{b} \mathrm{TOF}=\mathrm{g} \mathrm{PE} \mathrm{mol} \mathrm{Ni}{ }^{-1} \mathrm{~h}^{-1} .{ }^{c}$ Determined by GPC. ${ }^{d}$ Determined by NMR integration.

lengthened reaction time from 3-24 h, although causing the depletion of the activity, could increase the yields $(6.4$ and $13.2 \mathrm{~g}$, respectively) and $M_{n}$, but leave the PDI and branch number nearly unchanged, indicating the reasonable stability of the catalyst system at $25^{\circ} \mathrm{C}$. The activity increases with the ethylene pressure in the range of 14-28 bar in the entries 1-5. It suggests that the coordination of ethylene to the metal site ought to be crucial to the catalysis.

Entries 8-11 show that the ortho-substituted phenyl on the imino nitrogen can substantially help the activity. This is as expected and is consistent with the structure-to-reactivity relationship discovered in the diimine systems. ${ }^{3}$

Most intriguingly, the data of the entries 7 and 11-14 clearly exhibit that the less bulky amino substituents are favored by the ethylene polymerization. Further more, the activity of polymerization is rather susceptible to the conformational variation of the amino substituents. As shown in the entries 13 and 14, the catalysts of Ni-4c and $\mathrm{Ni}-\mathbf{5 c}$, which have the cyclic amino substituents afford much better activity than those with acyclic substituents of the comparable carbon numbers as shown in entries 9-11.

Such an independent but still cooperative structure-to-reactivity relationship from the unsymmetric aminoaldimine ligands is in contrast to the general understanding concluded from the symmetric diimine systems in which the ethylene coordination is not rate determining. ${ }^{3 \mathrm{a}-\mathrm{c}}$ In these amine-imine catalysts, although the ortho-substituents on the imino phenyl ring remains important, however, the polymerization activity appears to be more dependent to the amino substituents.

Unlike the symmetric diimine systems, the bidentate $\alpha$ aminoaldimines can lead to selective geometrical isomerism in square planar configuration. ${ }^{11}$ In the square-planar resting state, the polymer chain and ethylene monomer are considered to coordinate to the $\mathrm{Ni}$ (II) center, besides the auxiliary bidentate ligand. ${ }^{3 a, b}$ Concerning the stereoselectivity in the more stable trans form, the bulky polymer chain may be cis to the imino functionality; and the small ethylene is seated cis to the amine. This is because the Ni-N1-C21 angle (generally $>120^{\circ}$ ) around the imino $\mathrm{sp}^{3}$ nitrogen may accommodate greater steric tolerance than the $\mathrm{Ni}-\mathrm{N} 2-\mathrm{C}$ angles $\left(<110^{\circ}\right)$ around the amino $\mathrm{sp}^{3}$ nitrogen. Therefore, the bulkier amino substituents tend to destabilize the resting state by hampering the ethylene coordination.
Table 2 Selected parameters from calculations

\begin{tabular}{lcccc}
\hline Complex & cis-Ni-1b' $^{\prime}$ & trans-Ni-1b' $^{\prime}$ & cis-Ni-2b' & trans-Ni-2b' $^{\prime}$ \\
\hline$(\mathrm{Ni}-\mathrm{N} 1) / \AA$ & 2.201 & 2.085 & 2.106 & 2.162 \\
$(\mathrm{Ni}-\mathrm{N} 2) / \AA$ & 1.916 & 1.926 & 1.928 & 1.921 \\
$(\mathrm{C} 1-\mathrm{N} 1) / \AA$ & 1.278 & 1.278 & 1.276 & 1.279 \\
$(\mathrm{Ni}-\mathrm{C} 11) / \AA$ & 1.969 & 1.995 & 1.947 & 2.004 \\
$(\mathrm{Ni}-\mathrm{C} 14) / \AA$ & 2.034 & 2.063 & 2.035 & 2.073 \\
$(\mathrm{Ni}-\mathrm{C} 15) / \AA$ & 1.978 & 1.985 & 1.992 & 1.976 \\
$\mathrm{~N} 1-\mathrm{Ni}-\mathrm{N} 2{ }^{\circ}$ & 81.95 & 81.47 & 83.51 & 82.40 \\
Rel. $E / \mathrm{KJ} \mathrm{mol}^{-1}$ & 2.17 & 0 & 21.07 & 0 \\
\hline
\end{tabular}

\section{Calculation analysis}

Theoretical calculations also supports the assertion of steric concern. The relative stability for two geometrical isomers corresponding to $(\mathrm{L}-1 \mathrm{~b}) \mathrm{Ni}\left(\mathrm{C}_{2} \mathrm{H}_{4}\right)\left({ }^{n} \mathrm{Pr}\right)\left(\mathrm{Ni}-\mathbf{1 b}^{\prime}\right)$ and $(\mathrm{L}-2 \mathrm{~b}) \mathrm{Ni}\left(\mathrm{C}_{2} \mathrm{H}_{4}\right)\left({ }^{n} \mathrm{Pr}\right)(\mathrm{Ni}-$ $\mathbf{2}^{\prime}$ ) have been calculated. Some bonding data are listed in Table 2. The calculated structures are illustrated in Fig. 2. It is worthy of noting that the trans form is $2.17 \mathrm{~kJ} \mathrm{~mol}^{-1}$ more stable than the cis form for ( $\mathrm{L}-1 \mathrm{~b}) \mathrm{Ni}\left(\mathrm{C}_{2} \mathrm{H}_{4}\right)\left({ }^{n} \mathrm{Pr}\right)$. Whilst for $(\mathrm{L}-2 \mathrm{~b}) \mathrm{Ni}\left(\mathrm{C}_{2} \mathrm{H}_{4}\right)\left({ }^{n} \mathrm{Pr}\right)$, the trans form is $21.07 \mathrm{KJ} \mathrm{mol}^{-1}$ more stable than the cis derivative.
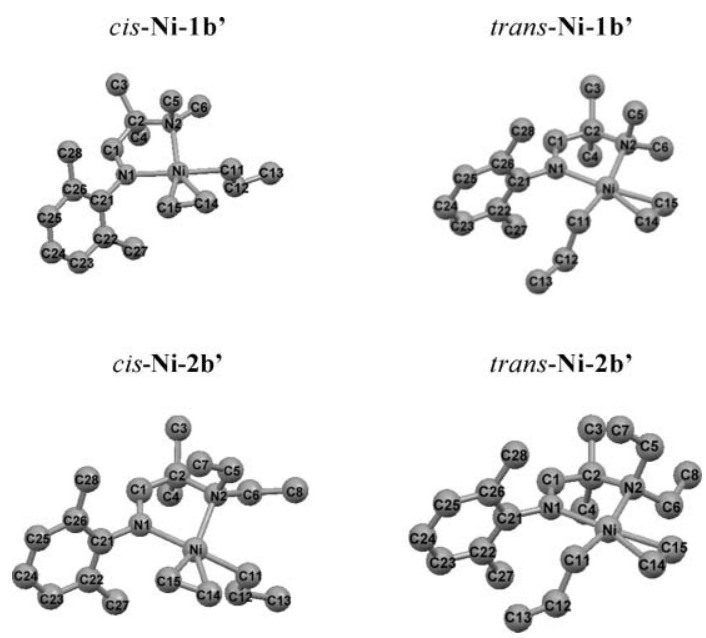

Fig. 2 Calculated structures for the geometrical isomers of $(\mathrm{L}-1 \mathrm{~b}) \mathrm{Ni}\left(\mathrm{C}_{2} \mathrm{H}_{4}\right)\left({ }^{n} \mathrm{Pr}\right)\left(\mathrm{Ni}-1 \mathbf{b}^{\prime}\right)$ and $(\mathrm{L}-2 \mathrm{~b}) \mathrm{Ni}\left(\mathrm{C}_{2} \mathrm{H}_{4}\right)\left({ }^{n} \mathrm{Pr}\right)\left(\mathrm{Ni}-2 \mathbf{b}^{\prime}\right)$. 
It is indicated that the geometrical isomerism in Ni-1 $\mathbf{b}^{\prime}$ probably makes little difference to ethylene polymerization. However in $\mathrm{Ni}$ $\mathbf{2} \mathbf{b}^{\prime}$, the steric hindrance resulting from the methyl groups on the $\mathrm{C} 2$ could cause the $\mathrm{N}$-bound ethyl groups to interfere the ethylene coordination, specifically in the trans configuration.

In addition, the steric hindrance between the two amino ethyl groups could enlarge the N1-Ni-N2 bite angle, which is known to be crucial to the polymerization activity. ${ }^{15}$ This can also explain why the small cyclic ring on the amino functionality can facilitate the ethylene polymerization.

\section{Concluding remarks}

In conclusion, the nickel catalysts bearing the amino and imino hybrid bidentate ligand are proved to afford a rare example wherein two coordinating functionalities confer the steric differentiation to the coordination that further resulting in the reactivity control to ethylene polymerization.

\section{Experimental}

\section{General procedure}

Commercially available reagents were purchased and used without further purification unless otherwise indicated. Toluene and diethyl ether were distilled from purple solutions of benzophenone ketyl under nitrogen and dichloromethane was dried over $\mathrm{P}_{2} \mathrm{O}_{5}$ and distilled immediately prior to use. Air-sensitive materials were manipulated under a nitrogen atmosphere in a glove box or by standard Schlenk techniques. The IR spectra were recorded on a Bio-Rad FTS-40 spectrophotometer. The NMR spectra were measured on a Bruker AC-300 or a Bruker AC-400 spectrometer. The corresponding frequencies for the ${ }^{13} \mathrm{C}$ NMR spectra were 75.469 $\mathrm{MHz}$ and 100.625 MHz, respectively. Values upfield of ${ }^{1} \mathrm{H}$ and ${ }^{13} \mathrm{C}$ data are given in $\delta(\mathrm{ppm})$ relative to tetramethylsilane ( $\delta 0.00)$ in $\mathrm{CDCl}_{3}$. Mass spectrometric analyses were collected on a JEOL SX-102A spectrometer. Elemental analysis was done on a Perkin-Elmer $2400 \mathrm{CHN}$ analyzer. Gel permeation chromatography (GPC) was performed in toluene at $25{ }^{\circ} \mathrm{C}$ using a Kratos model spectroflow 400 equipped with PL-mixed D exclusion limit 400k columns. Differential scanning calorimetry was measured under a continuous nitrogen purge $\left(20 \mathrm{~mL} \mathrm{~min}^{-1}\right)$ on a PerkinElmer Pyris 6 DSC instrument. The data were gathered on the third heating cycle using a heating and cooling scan rate of $10-15^{\circ} \mathrm{C} \mathrm{min}{ }^{-1}$. Thermogravimetric analysis was carried out using a TA Instruments TGA5100 with a hating rate of $10^{\circ} \mathrm{C} \mathrm{min}-1$ from $0-800{ }^{\circ} \mathrm{C}$ under a continuous nitrogen purge. Magnetic moments were measured between 2-300 K with an applied field up to $7 \mathrm{~T}$, using a MPMS7 SQUID magnetometer (Quantum Design, USA).

\section{Synthesis and characterization}

\section{$\mathrm{Me}_{2} \mathrm{NCMe}_{2} \mathrm{CH}=\mathrm{N}\left(2,6-\mathrm{Me}_{2} \mathrm{C}_{6} \mathrm{H}_{3}\right)(\mathrm{L}-1 \mathrm{~b})$}

$\mathrm{Me}_{2} \mathrm{NCMe}_{2} \mathrm{CHO}$ (4.6 g, $0.04 \mathrm{~mol}$ ) and 2,6-dimethylaniline (6.0 g, $0.05 \mathrm{mmol})$ were placed in $30 \mathrm{~mL}$ of toluene. Formic acid $(0.3 \mathrm{~mL}$ $99 \% \mathrm{v} / \mathrm{v}$ aqueous solution) was added, and the solution was refluxed in a set-up with a Dean-Stark trap for $1 \mathrm{~d}$. Toluene was removed in vacuo. The product was isolated by distillation to give a viscous yellow liquid in 58\% yield (5.06 g). IR ( $\mathrm{KBr}$ ) $v / \mathrm{cm}^{-1}: v_{\mathrm{C}=\mathrm{N}}$ 1662. ${ }^{1} \mathrm{H} \mathrm{NMR}\left(\mathrm{CDCl}_{3}, 400 \mathrm{MHz}\right) \delta / \mathrm{ppm}: 7.59$ $(\mathrm{s}, 1 \mathrm{H}, \mathrm{CH}=\mathrm{N}), 7.01-6.86\left(\mathrm{~m}, 3 \mathrm{H}, \mathrm{C}_{6} \mathrm{H}_{3}\right), 2.34\left(\mathrm{~s}, 6 \mathrm{H}, \mathrm{NCH}_{3}\right)$, $2.07\left(\mathrm{~s}, 6 \mathrm{H},\left(\mathrm{C}_{6} \mathrm{H}_{3}\right) \mathrm{CH}_{3}\right), 1.31\left(\mathrm{~s}, 6 \mathrm{H}, \mathrm{CCH}_{3}\right) .{ }^{13} \mathrm{C} \mathrm{NMR}\left(\mathrm{CDCl}_{3}\right.$, $100.625 \mathrm{MHz}) \delta / \mathrm{ppm}$ : $173.2(\mathrm{CH}=\mathrm{N}), 150.5,128-123$ (phenyl$C), 61.5\left(\mathrm{CCH}_{3}\right), 39.2\left(\mathrm{NCH}_{3}\right), 20.0\left(\mathrm{CCH}_{3}\right), 18.4\left(\left(\mathrm{C}_{6} \mathrm{H}_{3}\right) \mathrm{CH}_{3}\right)$; HR-FAB-MS $m / z$ : calcd for $\mathrm{C}_{14} \mathrm{H}_{23} \mathrm{~N}_{2} 219.1861$, found 219.1862 .

\section{$\mathrm{Me}_{2} \mathrm{NCMe}_{2} \mathrm{CH}=\mathrm{N}\left(2,6-{ }^{\mathrm{i}} \mathrm{Pr}_{2} \mathrm{C}_{6} \mathrm{H}_{3}\right)(\mathrm{L}-1 \mathrm{c})$}

Following the same procedure used for $\mathbf{L}-\mathbf{1 b}$, the reaction of $\mathrm{Me}_{2} \mathrm{NCMe}_{2} \mathrm{CHO}$ (4.6 g, $0.04 \mathrm{~mol}$ ) and 2,6- ${ }^{i} \mathrm{Pr}_{2} \mathrm{C}_{6} \mathrm{H}_{3} \mathrm{NH}_{2}(8.8 \mathrm{~g}$, $0.05 \mathrm{~mol}$ ) gave $\mathbf{L}-1 \mathbf{c}$ as a viscous yellow liquid in $47 \%$ yield $(11.5 \mathrm{~g})$. IR $(\mathrm{KBr}) v / \mathrm{cm}^{-1}: v_{\mathrm{C}=\mathrm{N}} 1626 .{ }^{1} \mathrm{H}$ NMR $\left(\mathrm{CDCl}_{3}, 300 \mathrm{MHz}\right) \delta / \mathrm{ppm}$ : $7.60(\mathrm{~s}, 1 \mathrm{H}, \mathrm{CH}=\mathrm{N}), 7.10-7.01\left(\mathrm{~m}, 3 \mathrm{H}, \mathrm{C}_{6} H_{3}\right), 2.90\left(\mathrm{~h}, J_{\mathrm{H}-\mathrm{H}}=\right.$ $6.9 \mathrm{~Hz}, 2 \mathrm{H},\left(\mathrm{CH}\left(\mathrm{CH}_{3}\right)_{2}\right), 2.35\left(\mathrm{~s}, 6 \mathrm{H}, \mathrm{NCH}_{3}\right), 1.32\left(\mathrm{~s}, 6 \mathrm{H}, \mathrm{CCH}_{3}\right)$, $1.15\left(\mathrm{~d}, J_{\mathrm{H}-\mathrm{H}}=6.9 \mathrm{~Hz}, 12 \mathrm{H}, \mathrm{CH}\left(\mathrm{CH}_{3}\right)_{2}\right) .{ }^{13} \mathrm{CNMR}\left(\mathrm{CDCl}_{3}\right.$, $100.625 \mathrm{MHz}) \delta / \mathrm{ppm}: 170.3(C \mathrm{H}=\mathrm{N}), 148.2-122.8$ (phenyl- $C$ ), $61.8\left(\mathrm{CCH}_{3}\right), 39.2\left(\mathrm{NCH}_{3}\right), 27.6\left(\mathrm{CH}\left(\mathrm{CH}_{3}\right)_{2}\right), 22.2\left(\mathrm{CCH}_{3}\right), 20.0$ $\left(\mathrm{CH}\left(\mathrm{CH}_{3}\right)_{2}\right)$; MS (FAB, $\left.\mathrm{m} / z\right)$ : $275.2\left(\mathrm{M}^{+}+1\right)$. Anal. calcd for $\mathrm{C}_{18} \mathrm{H}_{30} \mathrm{~N}_{2}$ : C 78.78, H 11.02, N 10.20. Found: C 77.73, H 11.19, $\mathrm{N} 9.31$.

\section{$\mathrm{Et}_{2} \mathrm{NCMe}_{2} \mathrm{CH}=\mathrm{NPh}(\mathrm{L}-2 \mathrm{a})$}

Following the same procedure used for $\mathbf{L}-\mathbf{1 b}$, the reaction of $\mathrm{Et}_{2} \mathrm{NCMe}_{2} \mathrm{CHO}(11.4 \mathrm{~g}, 0.08 \mathrm{~mol})$ and aniline $(7.4 \mathrm{~g}, 0.08$ mol) gave a viscous yellow liquid in $66 \%$ yield $(11.5 \mathrm{~g})$. IR (KBr) $v / \mathrm{cm}^{-1}: v_{\mathrm{C}=\mathrm{N}} 1648 .{ }^{1} \mathrm{H}$ NMR $\left(\mathrm{CDCl}_{3}, 300 \mathrm{MHz}\right) \delta / \mathrm{ppm}$ : $7.76(\mathrm{~s}, 1 \mathrm{H}, \mathrm{C} H=\mathrm{N}), 7.36-7.0\left(\mathrm{~m}, 5 \mathrm{H}, \mathrm{C}_{6} H_{5}\right), 2.70\left(\mathrm{q}, J_{\mathrm{H}-\mathrm{H}}=\right.$ $\left.7.1 \mathrm{~Hz}, 4 \mathrm{H}, \mathrm{NCH}_{2} \mathrm{CH}_{3}\right), 1.34\left(\mathrm{~s}, 6 \mathrm{H}, \mathrm{CCH}_{3}\right), 1.05\left(\mathrm{t}, J_{\mathrm{H}-\mathrm{H}}=\right.$ $\left.7.1 \mathrm{~Hz}, 6 \mathrm{H}, \mathrm{NCH}_{2} \mathrm{CH}_{3}\right) .{ }^{13} \mathrm{C} \mathrm{NMR}\left(\mathrm{CDCl}_{3}, 75.469 \mathrm{MHz}\right) \delta / \mathrm{ppm}$ : $172.6(C \mathrm{H}=\mathrm{N}), 151.8,130-112$ (phenyl- $C), 63.0\left(\mathrm{CCH}_{3}\right), 43.8$ $\left(\mathrm{NCH}_{2} \mathrm{CH}_{3}\right), 21.8\left(\mathrm{CCH}_{3}\right), 15.8\left(\mathrm{NCH}_{2} \mathrm{CH}_{3}\right)$. MS (FAB, $\left.m / z\right)$ : $219.2\left(\mathrm{M}^{+}+1\right)$. Anal. calcd for $\mathrm{C}_{14} \mathrm{H}_{22} \mathrm{~N}_{2}$ : C 77.01, H 10.16, N 12.83. Found: C 76.43, H 10.01, N 12.32 .

\section{$\mathrm{Et}_{2} \mathrm{NCMe}_{2} \mathrm{CH}=\mathrm{N}\left(2,6-\mathrm{Me}_{2} \mathrm{C}_{6} \mathrm{H}_{3}\right)(\mathrm{L}-2 \mathrm{~b})$}

Following the same procedure used for $\mathbf{L - 1 b}$, the reaction of $\mathrm{Et}_{2} \mathrm{NCMe}_{2} \mathrm{CHO}$ (10.0 g, $\left.0.07 \mathrm{~mol}\right)$ and 2,6- $\mathrm{Me}_{2} \mathrm{C}_{6} \mathrm{H}_{3} \mathrm{NH}_{2}(8.5 \mathrm{~g}$, $0.07 \mathrm{~mol}$ ) gave a viscous yellow liquid of $\mathbf{L}-\mathbf{2 b}$ in $50 \%$ yield (8.6 g). IR (KBr) $v / \mathrm{cm}^{-1}: v_{\mathrm{C}=\mathrm{N}} 1667 .{ }^{1} \mathrm{H} \mathrm{NMR}\left(\mathrm{CDCl}_{3}, 300 \mathrm{MHz}\right)$ $\delta /$ ppm: $7.58(\mathrm{~s}, 1 \mathrm{H}, \mathrm{CH}=\mathrm{N}), 7.01-6.88\left(\mathrm{~m}, 3 \mathrm{H}, \mathrm{C}_{6} H_{3}\right), 2.66$ (q, $\left.J_{\mathrm{H}-\mathrm{H}}=7.1 \mathrm{~Hz}, 4 \mathrm{H}, \mathrm{NCH}_{2} \mathrm{CH}_{3}\right), 2.07\left(\mathrm{~s}, 6 \mathrm{H},\left(\mathrm{C}_{6} \mathrm{H}_{3}\right) \mathrm{CH}_{3}\right), 1.34$ $\left(\mathrm{s}, 6 \mathrm{H}, \mathrm{CCH}_{3}\right), 1.06\left(\mathrm{t}, J_{\mathrm{H}-\mathrm{H}}=7.1 \mathrm{~Hz}, 6 \mathrm{H}, \mathrm{NCH}_{2} \mathrm{CH}_{3}\right) .{ }^{13} \mathrm{C}$ $\mathrm{NMR}\left(\mathrm{CDCl}_{3}, 75.469 \mathrm{MHz}\right) \delta / \mathrm{ppm}: 174.3(\mathrm{CH}=\mathrm{N}), 150.5,128-$ 123 (phenyl- $C$ ), $63.0\left(\mathrm{CCH}_{3}\right), 43.7\left(\mathrm{NCH}_{2} \mathrm{CH}_{3}\right), 22.1\left(\mathrm{CCH}_{3}\right)$, $18.3\left(\left(\mathrm{C}_{6} \mathrm{H}_{3}\right) \mathrm{CH}_{3}\right), 16.5\left(\mathrm{NCH}_{2} \mathrm{CH}_{3}\right)$. HR-FAB-MS: $m / z$ calcd for $\mathrm{C}_{16} \mathrm{H}_{27} \mathrm{~N}_{2}$ 247.2174, found 247.2175. Anal. calcd for $\mathrm{C}_{16} \mathrm{H}_{26} \mathrm{~N}_{2}: \mathrm{C}$ 78.00, H 10.64, N 11.37. Found: C 77.64, H 10.45, N 11.39.

\section{$\mathrm{Et}_{2} \mathrm{NCMe}_{2} \mathrm{CH}=\mathrm{N}\left(2,6-{ }^{i} \mathrm{Pr}_{2} \mathrm{C}_{6} \mathrm{H}_{3}\right)(\mathrm{L}-2 \mathrm{c})$}

Following the same procedure used for $\mathbf{L}-\mathbf{1 b}$, the reaction of $\mathrm{Et}_{2} \mathrm{NCMe}_{2} \mathrm{CHO}$ (10.0 g, $\left.0.07 \mathrm{~mol}\right)$ and $2,6{ }^{i} \mathrm{Pr}_{2} \mathrm{C}_{6} \mathrm{H}_{3} \mathrm{NH}_{2}(12.4 \mathrm{~g}$, $0.07 \mathrm{~mol}$ ) gave a viscous yellow liquid of $\mathbf{L - 2 \mathbf { c }}$ and was obtained in $44 \%$ yield $(9.3 \mathrm{~g})$. IR ( $\mathrm{KBr}) v / \mathrm{cm}^{-1}: v_{\mathrm{C}=\mathrm{N}} 1666 .{ }^{1} \mathrm{H} \mathrm{NMR}\left(\mathrm{CDCl}_{3}\right.$, $300 \mathrm{MHz}) \delta / \mathrm{ppm}: 7.56(\mathrm{~s}, 1 \mathrm{H}, \mathrm{CH}=\mathrm{N}), 7.09-7.05\left(\mathrm{~m}, 3 \mathrm{H}, \mathrm{C}_{6} H_{3}\right)$, $2.88\left(\mathrm{~h}, J_{\mathrm{H}-\mathrm{H}}=7.1 \mathrm{~Hz}, 1 \mathrm{H} \mathrm{CH}\left(\mathrm{CH}_{3}\right)_{2}\right) 2.65\left(\mathrm{q}, J_{\mathrm{H}-\mathrm{H}}=7.1 \mathrm{~Hz}\right.$, $\left.4 \mathrm{H}, \mathrm{NCH}_{2} \mathrm{CH}_{3}\right), 1.33\left(\mathrm{~s}, 6 \mathrm{H}, \mathrm{CCH}_{3}\right), 1.13\left(\mathrm{~d}, J_{\mathrm{H}-\mathrm{H}}=7.1 \mathrm{~Hz}\right.$, 
$\left.6 \mathrm{H}, \mathrm{CH}\left(\mathrm{CH}_{3}\right)_{2}\right), 1.05\left(\mathrm{t}, J_{\mathrm{H}-\mathrm{H}}=7.1 \mathrm{~Hz}, 6 \mathrm{H}, \mathrm{NCH}_{2} \mathrm{CH}_{3}\right) .{ }^{13} \mathrm{C}$ $\mathrm{NMR}\left(\mathrm{CDCl}_{3}, 75.469 \mathrm{MHz}\right) \delta / \mathrm{ppm}: 173.7(C \mathrm{H}=\mathrm{N}), 148.3,137.4$, 123.7, 122.8 (phenyl- $C$ ), $63.2\left(\mathrm{CCH}_{3}\right), 43.8\left(\mathrm{NCH}_{2} \mathrm{CH}_{3}\right), 27.6$ $\left(\mathrm{CH}\left(\mathrm{CH}_{3}\right)_{2}\right), 23.5\left(\mathrm{CH}\left(\mathrm{CH}_{3}\right)_{2}\right), 22.2\left(\mathrm{CCH}_{3}\right), 16.5\left(\mathrm{NCH}_{2} \mathrm{CH}_{3}\right)$. MS (FAB, $m / z)$ : $275.2\left(\mathrm{M}^{+}+1\right)$. Anal. calcd for $\mathrm{C}_{20} \mathrm{H}_{34} \mathrm{~N}_{2}: \mathrm{C}$ 79.41, H 11.32, N 9.26. Found: C 79.70, H 11.62, N 9.15.

\section{${ }^{n} \mathrm{Pr}_{2} \mathrm{NCMe}_{2} \mathrm{CH}=\mathrm{N}\left(2,6-{ }^{i} \mathrm{Pr}_{2} \mathrm{C}_{6} \mathrm{H}_{3}\right)(\mathrm{L}-3 \mathrm{c})$}

Following the same procedure used for $\mathbf{L}-\mathbf{1 b}$, the reaction of ${ }^{n} \mathrm{Pr}_{2} \mathrm{NCMe}_{2} \mathrm{CHO}$ (3.42 g, $0.02 \mathrm{~mol}$ ) and 2,6- ${ }^{i} \mathrm{Pr}_{2} \mathrm{C}_{6} \mathrm{H}_{3} \mathrm{NH}_{2}$ (3.55 g, $0.02 \mathrm{~mol}$ ) gave a yellow solid $\mathbf{L}-3 \mathbf{c}$ in $40 \%$ yield $(2.74 \mathrm{~g})$. IR $(\mathrm{KBr}) v / \mathrm{cm}^{-1}: v_{\mathrm{C}=\mathrm{N}} 1666 .{ }^{1} \mathrm{H}$ NMR $\left(\mathrm{CDCl}_{3}, 300 \mathrm{MHz}\right) \delta / \mathrm{ppm}$ : $7.59(\mathrm{~s}, 1 \mathrm{H}, \mathrm{CH}=\mathrm{N}), 7.12-7.04\left(\mathrm{~m}, 3 \mathrm{H}, \mathrm{C}_{6} H_{3}\right), 2.90\left(\mathrm{~h}, J_{\mathrm{H}-\mathrm{H}}=\right.$ $\left.6.7 \mathrm{~Hz}, 2 \mathrm{H}, \mathrm{CH}\left(\mathrm{CH}_{3}\right)_{2}\right), 2.51\left(\mathrm{~m}, 4 \mathrm{H}, \mathrm{CH}_{2} \mathrm{CH}_{2} \mathrm{CH}_{3}\right), 1.47(\mathrm{~m}$, $\left.J_{\mathrm{H}-\mathrm{H}}=7.2 \mathrm{~Hz}, 4 \mathrm{H}, \mathrm{NCH}_{2} \mathrm{CH}_{2} \mathrm{CH}_{3}\right), 1.33\left(\mathrm{~s}, 6 \mathrm{H}, \mathrm{CCH}_{3}\right), 1.60$ $\left(\mathrm{d}, J_{\mathrm{H}-\mathrm{H}}=6.7 \mathrm{~Hz}, 12 \mathrm{H}, \mathrm{CH}\left(\mathrm{CH}_{3}\right)_{2}\right) 0.85\left(\mathrm{t}, J_{\mathrm{H}-\mathrm{H}}=7.2 \mathrm{~Hz}, 6 \mathrm{H}\right.$, $\left.\mathrm{NCH}_{2} \mathrm{CH}_{2} \mathrm{CH}_{3}\right) .{ }^{13} \mathrm{C} \mathrm{NMR}\left(\mathrm{CDCl}_{3}, 75.469 \mathrm{MHz}\right) \delta / \mathrm{ppm}: 173.8$ $(C \mathrm{H}=\mathrm{N}), 148.3,137.4,123.7,122.8$ (phenyl- $C), 63.1\left(C \mathrm{CH}_{3}\right), 53.3$ $\left(\mathrm{NCH}_{2} \mathrm{CH}_{2} \mathrm{CH}_{3}\right), 27.5\left(\mathrm{CH}\left(\mathrm{CH}_{3}\right)_{2}\right), 24.5\left(\mathrm{NCH}_{2} \mathrm{CH}_{2} \mathrm{CH}_{3}\right), 23.5$ $\left(\mathrm{CH}\left(\mathrm{CH}_{3}\right)_{2}\right), 22.1\left(\mathrm{CCH}_{3}\right), 11.7\left(\mathrm{NCH}_{2} \mathrm{CH}_{2} \mathrm{CH}_{3}\right)$. MS (FAB, $\left.m / z\right)$ : 329.3 $\left(\mathrm{M}^{+}+1\right)$. Anal. calcd for $\mathrm{C}_{22} \mathrm{H}_{38} \mathrm{~N}_{2}$ : C 79.95, H 11.59, N 8.47. Found: C 79.40, H 11.60, N 8.24.

\section{$\left(c-\mathrm{C}_{3} \mathrm{H}_{6}\right) \mathrm{NCMe}_{2} \mathrm{CH}=\mathrm{N}\left(2,6^{-}{ }^{i} \operatorname{Pr}_{2} \mathrm{C}_{6} \mathrm{H}_{3}\right)(\mathrm{L}-4 \mathrm{c})$}

Following the same procedure used for $\mathbf{L}-\mathbf{1 b}$, the reaction of $c-\mathrm{C}_{3} \mathrm{H}_{6} \mathrm{NCMe}_{2} \mathrm{CHO}$ (5.0 g, $\left.0.04 \mathrm{~mol}\right)$ and 2,6- ${ }^{i} \mathrm{Pr}_{2} \mathrm{C}_{6} \mathrm{H}_{3} \mathrm{NH}_{2}(8.8 \mathrm{~g}$, $0.05 \mathrm{~mol}$ ) gave the product $\mathbf{L}-4 \mathbf{c}$. Isolation gives a yellow liquid in $47 \%$ yield $(5.4 \mathrm{~g}) .{ }^{1} \mathrm{H}$ NMR $\left(\mathrm{CDCl}_{3}, 300 \mathrm{MHz}\right) \delta / \mathrm{ppm}: 7.60$ (s, $1 \mathrm{H}, \mathrm{CH}=\mathrm{N}), 7.05\left(\mathrm{~m}, 3 \mathrm{H}, \mathrm{C}_{6} H_{3}\right), 3.39\left(\mathrm{t}, J_{\mathrm{H}-\mathrm{H}}=7.2 \mathrm{~Hz}, 4 \mathrm{H}\right.$, $\left.\mathrm{N}\left(\mathrm{CH}_{2}\right)_{2} \mathrm{CH}_{2}\right), 2.87\left(\mathrm{~m}, J_{\mathrm{H}-\mathrm{H}}=6.7 \mathrm{~Hz}, 4 \mathrm{H}, \mathrm{CH}\left(\mathrm{CH}_{3}\right)_{2}\right), 2.05(\mathrm{~m}$, $\left.J_{\mathrm{H}-\mathrm{H}}=7.2 \mathrm{~Hz}, 2 \mathrm{H}, \mathrm{N}\left(\mathrm{CH}_{2}\right)_{2} \mathrm{CH}_{2}\right), 1.22\left(\mathrm{~s}, \mathrm{H}, \mathrm{C}\left(\mathrm{CH}_{3}\right)_{2}\right), 1.13$ $\left(\mathrm{d}, J_{\mathrm{H}-\mathrm{H}}=6.7 \mathrm{~Hz}, 12 \mathrm{H}, \mathrm{CH}\left(\mathrm{CH}_{3}\right)_{2}\right) \cdot{ }^{13} \mathrm{C} \mathrm{NMR}\left(\mathrm{CDCl}_{3}, 75.469\right.$ MHz) $\delta /$ ppm: $170.8(C H=N), 137.2,123.9,122.8$ (phenyl- $C), 59.1$ $\left(\mathrm{CCH}_{3}\right), 47.6\left(\mathrm{NCH}_{2}\right), 27.6\left(\mathrm{CH}\left(\mathrm{CH}_{3}\right)_{2}\right), 23.4\left(\mathrm{CH}\left(\mathrm{CH}_{3}\right)_{2}\right), 20.0$ $\left(\mathrm{CCH}_{3}\right), 16.5\left(\mathrm{CH}_{2}\left(\mathrm{CH}_{2}\right)_{2}\right)$.

\section{$\left(c-\mathrm{C}_{4} \mathrm{H}_{8}\right) \mathrm{NCMe}_{2} \mathrm{CH}=\mathrm{N}\left(2,6-{ }^{i} \mathrm{Pr}_{2} \mathrm{C}_{6} \mathrm{H}_{3}\right)(\mathrm{L}-5 \mathrm{c})$}

Following the same procedure used for $\mathbf{L}-\mathbf{1 b}$, the reaction of $c-\mathrm{C}_{4} \mathrm{H}_{8} \mathrm{NCMe}_{2} \mathrm{CHO}\left(10.0 \mathrm{~g}, 0.07 \mathrm{~mol}\right.$ ) and $2,6-{ }^{i} \mathrm{Pr}_{2} \mathrm{C}_{6} \mathrm{H}_{3} \mathrm{NH}_{2}$ $(14.1 \mathrm{~g}, 0.08 \mathrm{~mol})$ gave the product L-5c. Isolation was done by crystallization to give a yellow solid in $50 \%$ yield $(10.5 \mathrm{~g})$. IR $(\mathrm{KBr}) v / \mathrm{cm}^{-1}: v_{\mathrm{C}=\mathrm{N}} 1666 .{ }^{1} \mathrm{H} \mathrm{NMR}\left(\mathrm{CDCl}_{3}, 300 \mathrm{MHz}\right)$ $\delta /$ ppm: $7.67(\mathrm{~s}, 1 \mathrm{H}, \mathrm{CH}=\mathrm{N}), 7.10-7.01\left(\mathrm{~m}, 3 \mathrm{H}, \mathrm{C}_{6} H_{3}\right), 2.89(\mathrm{~h}$, $\left.2 \mathrm{H}, J_{\mathrm{H}-\mathrm{H}}=7.1 \mathrm{~Hz}, 2 \mathrm{H}, \mathrm{CH}\left(\mathrm{CH}_{3}\right)_{2}\right), 2.78\left(\mathrm{~m}, 4 \mathrm{H}, \mathrm{NCH}_{2} \mathrm{CH}_{2}\right)$, $1.77\left(\mathrm{~m}, 4 \mathrm{H}, \mathrm{NCH}_{2} \mathrm{CH}_{2}\right), 1.38\left(\mathrm{~s}, 6 \mathrm{H}, \mathrm{CCH}_{3}\right), 1.13\left(\mathrm{~d}, J_{\mathrm{H}-\mathrm{H}}=\right.$ $\left.7.1 \mathrm{~Hz}, 12 \mathrm{H}, \mathrm{CH}\left(\mathrm{CH}_{3}\right)_{2}\right) .{ }^{13} \mathrm{C} \mathrm{NMR}\left(\mathrm{CDCl}_{3}, 75.469 \mathrm{MHz}\right)$ $\delta /$ ppm: $172.1(C \mathrm{H}=\mathrm{N}), 148.3,137.4-122.8$ (phenyl- $C$ ), 59.7 $\left(\mathrm{CCH}_{3}\right), 46.5\left(\mathrm{NCH}_{2} \mathrm{CH}_{2}\right), 27.6\left(\mathrm{CH}\left(\mathrm{CH}_{3}\right)_{2}\right), 24.1\left(\mathrm{NCH}_{2} \mathrm{CH}_{2}\right)$, $23.5\left(\mathrm{CH}\left(\mathrm{CH}_{3}\right)_{2}\right), 21.8\left(\mathrm{CCH}_{3}\right)$. MS (FAB, $\left.m / z\right): 301.2\left(\mathrm{M}^{+}+1\right)$. Anal. calcd for $\mathrm{C}_{20} \mathrm{H}_{32} \mathrm{~N}_{2}$ : C 79.94, $\mathrm{H} \mathrm{10.73,} \mathrm{N} \mathrm{9.32.} \mathrm{Found:} \mathrm{C}$ 80.32, H 11.12, N 9.27.

\section{$\left[\mathrm{Me}_{2} \mathrm{NCMe}_{2} \mathrm{C}=\mathrm{N}\left(2,6-\mathrm{Me}_{2} \mathrm{C}_{6} \mathrm{H}_{3}\right)\right] \mathrm{NiBr}_{2}(\mathrm{Ni}-1 \mathrm{~b})$}

(DME)NiBr 2 (300 mg, $1.0 \mathrm{mmol})$ and L-1b (327 mg, $1.5 \mathrm{mmol})$ were placed in a round-bottomed flask under nitrogen. Pre-dried $\mathrm{CH}_{2} \mathrm{Cl}_{2}(15 \mathrm{~mL})$ was transferred in vacuo. The orange solution turned to violet within $10 \mathrm{~min}$. The reaction was allowed to complete at $25{ }^{\circ} \mathrm{C}$. After removal of the supernatant solid, the reaction solution was concentrated. The addition of dry $\mathrm{Et}_{2} \mathrm{O}$ resulted in the solid product, and the yield of $\mathbf{N i - 1 b}$ was $74 \%$ (321 mg) after recrystallization from $\mathrm{CH}_{2} \mathrm{Cl}_{2}-\mathrm{Et}_{2} \mathrm{O}$. MS (FAB, $\mathrm{m} / z$ ): $355.0\left(\mathrm{M}^{+}-\mathrm{Br}\right)$. Anal. calcd for $\mathrm{C}_{14} \mathrm{H}_{22} \mathrm{~N}_{2} \mathrm{Br}_{2} \mathrm{Ni}$ : C 38.49, H 5.08, N 6.41. Found: C 38.57, H 5.06, N 6.04. Crystals suitable for X-ray diffraction experiment were obtained by slow diffusion of diethyl ether into a saturated dichloromethane solution of Ni-1b.

\section{$\left[\mathrm{Me}_{2} \mathrm{NCMe}_{2} \mathrm{C}=\mathrm{N}\left(2,6-{ }^{i} \mathrm{Pr}_{2} \mathrm{C}_{6} \mathrm{H}_{3}\right)\right] \mathrm{NiBr}_{2}(\mathrm{Ni}-1 \mathrm{c})$}

Following the same procedure used for $\mathbf{N i - 1 b}$, the reaction of (DME)NiBr 2 (100 mg, $0.32 \mathrm{mmol})$ and L-1c (134 mg, $0.49 \mathrm{mmol})$ gave violet solid of Ni-1c in $57 \%$ yield $(92 \mathrm{mg})$. MS (FAB, $\mathrm{m} / z$ ): $411.1\left(\mathrm{M}^{+}+1-\mathrm{Br}\right)$. Anal. calcd for $\mathrm{C}_{18} \mathrm{H}_{30} \mathrm{~N}_{2} \mathrm{Br}_{2} \mathrm{Ni}$ : C 43.86, $\mathrm{H}$ 6.11, N 5.68. Found: C 43.68, H 6.12, N 5.34.

\section{$\left[\mathrm{Et}_{2} \mathrm{NCMe}_{2} \mathrm{CH}=\mathrm{NPh}\right] \mathrm{NiBr}_{2}(\mathrm{Ni}-2 \mathrm{a})$}

Following the same procedure used for Ni-1b, the reaction of (DME)NiBr 2 (300 mg, $1.0 \mathrm{mmol})$ and L-2a (327 mg, $1.5 \mathrm{mmol})$ gave a violet solid Ni-2a in $62 \%$ yield $(264 \mathrm{mg}) .{ }^{1} \mathrm{H} \mathrm{NMR}\left(\mathrm{CDCl}_{3}\right.$, $300 \mathrm{MHz}) \delta / \mathrm{ppm}: 8.56(\mathrm{~s}, 1 \mathrm{H}, \mathrm{CH}=\mathrm{N}), 7.44-7.18(\mathrm{~m}, 5 \mathrm{H}$, phenyl$H$ ), 3.37, $2.96\left(\mathrm{~m}, \mathrm{~m}, 2 \mathrm{H}, 2 \mathrm{H}, \mathrm{NCH}_{2} \mathrm{CH}_{3}\right), 1.74$ (s, 6H, $\mathrm{CCH}_{3}$ ), $1.59\left(\mathrm{t}, J_{\mathrm{H}-\mathrm{H}}=7.1 \mathrm{~Hz}, 6 \mathrm{H}, \mathrm{NCH}_{2} \mathrm{CH}_{3}\right) \cdot{ }^{13} \mathrm{C} \mathrm{NMR}\left(\mathrm{CDCl}_{3}\right.$, $75.469 \mathrm{MHz}) \delta / \mathrm{ppm}: 161.1(\mathrm{CH}=\mathrm{N}), 148.4,128.8,126.9,120.8$ (phenyl- $\mathrm{C}$ ), $69.0\left(\mathrm{CCH}_{3}\right), 46.2\left(\mathrm{NCH}_{2} \mathrm{CH}_{3}\right), 21.2\left(\mathrm{CCH}_{3}\right), 11.8$ $\left(\mathrm{NCH}_{2} \mathrm{CH}_{3}\right)$. MS (FAB, $\left.m / z\right)$ : $355.0\left(\mathrm{M}^{+}+1-\mathrm{Br}\right)$. Anal. calcd for $\mathrm{C}_{14} \mathrm{H}_{22} \mathrm{Br}_{2} \mathrm{~N}_{2} \mathrm{Ni}$ : C 38.49, H 5.08, N 6.41. Found: C 38.78, H 5.03, N 6.37.

\section{$\left[\mathrm{Et}_{2} \mathrm{NCMe}_{2} \mathrm{C}=\mathrm{N}\left(2,6-\mathrm{Me}_{2} \mathrm{C}_{6} \mathrm{H}_{3}\right)\right] \mathrm{NiBr}_{2}(\mathrm{Ni}-2 \mathrm{~b})$}

Following the same procedure used for Ni-1b, the reaction of (DME) $\mathrm{NiBr}_{2}(300 \mathrm{mg}, 1.0 \mathrm{mmol})$ and $\mathbf{L}-2 \mathbf{b}(358 \mathrm{mg}, 1.5 \mathrm{mmol})$ gave a violet solid of $\mathbf{N i - 2 b}$ in $57 \%$ yield $(264 \mathrm{mg}) .{ }^{1} \mathrm{H}$ NMR $\left(\mathrm{CDCl}_{3}, 300 \mathrm{MHz}\right) \delta / \mathrm{ppm}: 8.29(\mathrm{~s}, 1 \mathrm{H}, \mathrm{CH}=\mathrm{N}), 7.06-6.98(\mathrm{~m}, 3 \mathrm{H}$, phenyl- $H$ ), 3.45, $3.07\left(\mathrm{~m}, \mathrm{~m} J_{\mathrm{H}-\mathrm{H}}=7.1 \mathrm{~Hz}, 2 \mathrm{H}, 2 \mathrm{H}, \mathrm{NCH}_{2} \mathrm{CH}_{3}\right)$, $2.10\left(\mathrm{~s}, 6 \mathrm{H},\left(\mathrm{C}_{6} \mathrm{H}_{3}\right) \mathrm{CH}_{3}\right), 1.83\left(\mathrm{~s}, 6 \mathrm{H}, \mathrm{CCH}_{3}\right), 1.70\left(\mathrm{t}, J_{\mathrm{H}-\mathrm{H}}\right.$ $\left.=7.1 \mathrm{~Hz}, 6 \mathrm{H}, \mathrm{NCH}_{2} \mathrm{CH}_{3}\right) \cdot{ }^{13} \mathrm{C} \mathrm{NMR}\left(\mathrm{CDCl}_{3}, 75.469 \mathrm{MHz}\right)$ $\delta /$ ppm: $164.4(C \mathrm{H}=\mathrm{N}), 147.9,127.9,125.6,124.1$, (phenyl- $C$ ), $69.8\left(\mathrm{CCH}_{3}\right), 46.6\left(\mathrm{NCH}_{2} \mathrm{CH}_{3}\right), 21.4\left(\mathrm{CCH}_{3}\right), 18.3\left(\left(\mathrm{C}_{6} \mathrm{H}_{3}\right) \mathrm{CH}_{3}\right)$, $11.9\left(\mathrm{NCH}_{2} \mathrm{CH}_{3}\right)$. MS (FAB, $\left.m / z\right)$ : $383.1\left(\mathrm{M}^{+}-\mathrm{Br}\right)$. Anal. calcd for $\mathrm{C}_{16} \mathrm{H}_{26} \mathrm{Br}_{2} \mathrm{~N}_{2} \mathrm{Ni} \cdot 0.324 \mathrm{CH}_{2} \mathrm{Cl}_{2}$ : C 45.92, H 6.41, N 6.03. Found: C 45.92, H 6.40, N 6.48.

\section{$\left[\mathrm{Et}_{2} \mathrm{NCMe}_{2} \mathrm{C}=\mathrm{N}\left(2,6^{i}{ }^{i} \mathrm{Pr}_{2} \mathrm{C}_{6} \mathrm{H}_{3}\right)\right] \mathrm{NiBr}_{2}(\mathrm{Ni}-2 \mathrm{c})$}

Following the same procedure used for $\mathbf{N i - 1 b}$, the reaction of (DME)NiBr 2 (300 mg, $1.0 \mathrm{mmol})$ and L-2c (453 mg, $1.5 \mathrm{mmol})$ gave violet $\mathbf{N i - 2 c}$ in $56 \%$ yield $(291 \mathrm{mg}) .{ }^{1} \mathrm{H} \mathrm{NMR}\left(\mathrm{CDCl}_{3}, 300\right.$ $\mathrm{MHz}) \delta / \mathrm{ppm}: 8.29(\mathrm{~s}, 1 \mathrm{H}, \mathrm{CH}=\mathrm{N}), 7.14$ (br, 3H, phenyl- $H$ ), $3.49,3.12$ (br, 2H, 2H, $\left.\mathrm{NCH}_{2} \mathrm{CH}_{3}\right), 2.73\left(\mathrm{br}, 1 \mathrm{H}, \mathrm{CH}\left(\mathrm{CH}_{3}\right)_{2}\right)$ ), 1.85 (s, 6H, $\left.\mathrm{CCH}_{3}\right), 1.74$ (br, 6H, $\mathrm{NCH}_{2} \mathrm{CH}_{3}$ ), 1.19 (br, 6H, $\left.\left.\mathrm{CH}\left(\mathrm{CH}_{3}\right)_{2}\right)\right) .{ }^{13} \mathrm{C} \mathrm{NMR}\left(\mathrm{CDCl}_{3}, 75.469 \mathrm{MHz}\right) \delta / \mathrm{ppm}: 163.9$ $(C \mathrm{H}=\mathrm{N}), 150.3,136.0,124.5122 .5$ (phenyl- $C), 70.0\left(\mathrm{CCH}_{3}\right), 46.6$ $\left(\mathrm{NCH}_{2} \mathrm{CH}_{3}\right), 27.5\left(\mathrm{CH}\left(\mathrm{CH}_{3}\right)_{2}\right), 23.1\left(\mathrm{CH}\left(\mathrm{CH}_{3}\right)_{2}\right), 21.4\left(\mathrm{CCH}_{3}\right)$, $12.0\left(\mathrm{NCH}_{2} \mathrm{CH}_{3}\right)$. MS (FAB, $\left.m / z\right)$ : $439.1\left(\mathrm{M}^{+}-\mathrm{Br}\right)$. Anal. calcd 
Table 3 X-Ray crystal parameters and data collection

\begin{tabular}{|c|c|c|c|c|c|c|}
\hline Compound & Ni-1b & Ni-1c & Ni-2a & $\mathrm{Ni}-\mathbf{2 b}$ & $\mathrm{Ni}-4 \mathrm{c}$ & $\mathrm{Ni}-5 \mathrm{c}$ \\
\hline Formula & $\mathrm{C}_{14} \mathrm{H}_{22} \mathrm{Br}_{2} \mathrm{~N}_{2} \mathrm{Ni}$ & $\mathrm{C}_{18} \mathrm{H}_{30} \mathrm{Br}_{2} \mathrm{~N}_{2} \mathrm{Ni}$ & $\mathrm{C}_{14} \mathrm{H}_{22} \mathrm{Br}_{2} \mathrm{~N}_{2} \mathrm{Ni}$ & $\mathrm{C}_{16} \mathrm{H}_{26} \mathrm{Br}_{2} \mathrm{~N}_{2} \mathrm{Ni}$ & $\mathrm{C}_{19} \mathrm{H}_{30} \mathrm{Br}_{2} \mathrm{~N}_{2} \mathrm{Ni}$ & $\mathrm{C}_{20} \mathrm{H}_{32} \mathrm{Br}_{2} \mathrm{~N}_{2} \mathrm{Ni}$ \\
\hline$M_{\mathrm{r}} / \mathrm{g} \mathrm{mol}^{-1}$ & 436.87 & 492.97 & 436.87 & 464.92 & 504.98 & 519.01 \\
\hline Crystal size/mm & $0.25 \times 0.20 \times 0.15$ & $0.25 \times 0.20 \times 0.15$ & $0.20 \times 0.20 \times 0.15$ & $0.20 \times 0.10 \times 0.03$ & $0.25 \times 0.20 \times 0.15$ & $0.25 \times 0.20 \times 0.15$ \\
\hline Crystal system & Monoclinic & Orthorhombic & Monoclinic & Triclinic & Orthorhombic & Monoclinic \\
\hline Space group & $P 2_{1} / c$ & $\operatorname{Pna2}_{1}$ & $P 2_{1} / c$ & $P \overline{1}$ & Pna2 $_{1}$ & $P 2_{1} / c$ \\
\hline$a / \AA ̊$ & $17.3020(4)$ & $15.225(3)$ & $7.3858(3)$ & $7.9039(3)$ & $15.1090(3)$ & $8.66900(10)$ \\
\hline$b / \AA ̊$ & $13.8610(3)$ & $10.217(2)$ & $14.1749(4)$ & $8.6754(3)$ & $10.4250(3)$ & $14.1770(2)$ \\
\hline$c / \AA ̊$ & $15.2240(3)$ & $14.312(3)$ & $16.5776(6)$ & $15.4932(4)$ & $14.4930(3)$ & $19.0860(3)$ \\
\hline$\alpha /{ }^{\circ}$ & 90 & 90 & 90 & $102.012(1)$ & 90 & 90 \\
\hline$\beta /^{\circ}$ & $108.879(2)$ & 90 & $95.731(1)$ & $95.157(1)$ & 90 & $101.9170(10)$ \\
\hline$\gamma /{ }^{\circ}$ & 90 & 90 & 90 & $113.542(1)$ & 90 & 90 \\
\hline$V / \AA^{3}$ & $3454.65(13)$ & $2226.3(8)$ & $1726.85(11)$ & $934.71(6)$ & $2282.81(9)$ & $2295.12(6)$ \\
\hline$Z$ & 8 & 4 & 4 & 2 & 4 & 4 \\
\hline$\rho_{\text {calcd }} / \mathrm{Mg} \mathrm{m}^{-3}$ & 1.680 & 1.471 & 1.680 & 1.652 & 1.469 & 1.502 \\
\hline$F(000)$ & 1744 & 1000 & 872 & 468 & 1024 & 1056 \\
\hline$T / \mathrm{K}$ & $295(2)$ & $295(2)$ & $150(1)$ & $150(2)$ & $295(2)$ & $295(2)$ \\
\hline$\mu / \mathrm{mm}^{-1}$ & 5.739 & 4.462 & 5.741 & 5.308 & 4.354 & 4.333 \\
\hline Transmission & $0.260-0.446$ & $0.403-0.519$ & $0.3691-0.4921$ & $0.4790-0.6944$ & $0.338-0.533$ & $0.325-0.534$ \\
\hline$\theta$ range $/^{\circ}$ & $1.24-27.47$ & $2.40-27.47$ & $1.89-27.50$ & $2.64-27.50$ & $2.73-27.49$ & $3.75-27.49$ \\
\hline$h, k, l$ & & $-16-19,-12-13, \pm 18$ & \pm & & & \\
\hline & $22, \pm 17, \pm 19$ & & $9,-18-16,-19-21$ & $10, \pm 11, \pm 20$ & $19, \pm 13, \pm 18$ & $11, \pm 18,-23-24$ \\
\hline Reflections collected & 26721 & 12969 & 10220 & 10492 & 13320 & 17428 \\
\hline $\begin{array}{l}\text { Independent } \\
\text { reflections }\end{array}$ & 7918 & 4515 & 3919 & 4155 & 4895 & 5242 \\
\hline$R_{\text {int }}$ & 0.0970 & 0.0479 & 0.0371 & 0.0420 & 0.0484 & 0.0456 \\
\hline Data/restraints & $7918 / 3$ & $4515 / 1$ & $3919 / 0$ & $4155 / 0$ & $4895 / 1$ & $5242 / 0$ \\
\hline Parameters & 335 & 209 & 176 & 196 & 218 & 227 \\
\hline$R_{1}[I>2 \sigma(I)]$ & 0.0942 & 0.0583 & 0.0506 & 0.0451 & 0.0436 & 0.0408 \\
\hline $\mathrm{w} R_{2}[I>2 \sigma(I)]$ & 0.2648 & 0.1458 & 0.1113 & 0.0785 & 0.0974 & 0.1046 \\
\hline$R_{1}$ (all data) & 0.1728 & 0.1057 & 0.0680 & 0.0687 & 0.0814 & 0.0649 \\
\hline $\mathrm{w} R_{2}$ (all data) & 0.2911 & 0.1713 & 0.1185 & 0.0853 & 0.1141 & 0.1210 \\
\hline Goodness of fit on $F^{2}$ & 1.543 & 1.023 & 1.092 & 1.057 & 0.994 & 1.025 \\
\hline $\begin{array}{l}\text { Largest diffraction } \\
\text { peak and hole, e } \AA^{-3}\end{array}$ & 1.518 and -0.939 & 0.698 and -0.549 & 0.727 and -1.442 & 0.473 and -0.634 & 0.585 and -0.465 & 0.467 and -0.559 \\
\hline
\end{tabular}

for $\mathrm{C}_{20} \mathrm{H}_{34} \mathrm{~N}_{2} \mathrm{Br}_{2} \mathrm{Ni}$ : C 46.11, $\mathrm{H}$ 6.58, N 5.38. Found: C 46.33, $\mathrm{H}$ 6.47, N 5.11 .

\section{$\left[{ }^{n} \mathrm{Pr}_{2} \mathrm{NCMe}_{2} \mathrm{C}=\mathrm{N}\left(2,6{ }^{i}{ }^{i} \mathrm{Pr}_{2} \mathrm{C}_{6} \mathrm{H}_{3}\right)\right] \mathrm{NiBr}_{2}(\mathrm{Ni}-3 \mathrm{c})$}

Following the same procedure used for $\mathbf{N i - 1 b}$, the reaction of (DME)NiBr 2 (100 mg, $0.32 \mathrm{mmol})$ and L-3c (160 mg, $0.49 \mathrm{mmol})$ gave violet Ni-3c in $40 \%$ yield $(72 \mathrm{mg})$. MS (FAB, $\mathrm{m} / \mathrm{z})$ : $413\left(\mathrm{M}^{+}-\right.$ $\mathrm{Br})$. UV-vis, $\lambda_{\max } / \mathrm{nm}\left(\varepsilon / \mathrm{M}^{-1} \mathrm{~cm}^{-1}\right): 515$ (22.4). Anal. calcd for $\mathrm{C}_{22} \mathrm{H}_{38} \mathrm{~N}_{2} \mathrm{Br}_{2} \mathrm{Ni}$ : C 48.13, H 6.98, N 5.10. Found: C 48.78, H 7.51, N 4.71 .

\section{$\left[\left(c-\mathrm{C}_{3} \mathrm{H}_{6}\right) \mathrm{NCMe}_{2} \mathrm{C}=\mathrm{N}\left(2,6-{ }^{i} \mathrm{Pr}_{2} \mathrm{C}_{6} \mathrm{H}_{3}\right)\right] \mathrm{NiBr}_{2}(\mathrm{Ni}-4 \mathrm{c})$}

Following the same procedure used for $\mathbf{N i - 1 b}$, the reaction of (DME) $\mathrm{NiBr}_{2}$ (100 mg, $\left.0.32 \mathrm{mmol}\right)$ and L-4c (140 mg, $\left.0.49 \mathrm{mmol}\right)$ gave violet Ni-4c in $42 \%$ yield (48 mg). MS (FAB, $\mathrm{m} / z$ ): 425.1 $\left(\mathrm{M}^{+}-\mathrm{Br}\right)$. UV-vis, $\lambda_{\max } / \mathrm{nm}\left(\varepsilon / \mathrm{M}^{-1} \mathrm{~cm}^{-1}\right): 517$ (15.5). Anal. calcd for $\mathrm{C}_{19} \mathrm{H}_{30} \mathrm{~N}_{2} \mathrm{Br}_{2} \mathrm{Ni}$ : C 45.19, H 5.99, N 5.55. Found: C 44.37, H 5.77, N 5.28.

\section{$\left[\left(c-\mathrm{C}_{4} \mathrm{H}_{8}\right) \mathrm{NCMe}_{2} \mathrm{C}=\mathrm{N}\left(2,6-{ }^{i} \mathrm{Pr}_{2} \mathrm{C}_{6} \mathrm{H}_{3}\right)\right] \mathrm{NiBr}_{2}(\mathrm{Ni}-5 \mathrm{c})$}

Following the same procedure used for $\mathbf{N i - 1 b}$, the reaction of (DME) $\mathrm{NiBr}_{2}$ (100 mg, $0.32 \mathrm{mmol}$ ) and L-5c (150 mg, $\left.0.49 \mathrm{mmol}\right)$ gave violet Ni-5c in 53\% yield (52 mg). MS (FAB, $\mathrm{m} / \mathrm{z})$ : $413\left(\mathrm{M}^{+}-\right.$
Br). UV-vis, $\lambda_{\max } / \mathrm{nm}\left(\varepsilon / \mathrm{M}^{-1} \mathrm{~cm}^{-1}\right): 519$ (107). Anal. calcd for $\mathrm{C}_{20} \mathrm{H}_{32} \mathrm{~N}_{2} \mathrm{Br}_{2} \mathrm{Ni}$ : C 46.29, H 6.21, N 5.40. Found: C 45.93, H 6.12, N 5.20.

\section{General procedure for polymerization of ethylene}

Into a $600 \mathrm{~mL}$ Parr autoclave was placed the nickel complexes $(22-42 \mathrm{mg})$ and MAO $(6-8 \mathrm{~mL})$ in dried toluene $(100 \mathrm{~mL})$. The autoclave was sealed. Upon flushing with ethylene gas several times, the ethylene gas was pressurized. During the reaction, ethylene was refilled when the pressure was found to drop. The mixture was stirred for a period of time. The reaction was quenched by venting the autoclave followed by the addition of methanol- $\mathrm{HCl}(4: 1)$. The precipitated polymers were filtered from solution and dried in vacuo.

In a typical run, to a $600 \mathrm{~mL}$ autoclave was placed $22 \mathrm{mg}$ of the catalyst and $6 \mathrm{~mL}$ MAO $(10 \mathrm{wt} \%)$ in $100 \mathrm{~mL}$ of pre-dried toluene. The thermostated autoclave was sealed and flushed several times with ethylene. The ethylene was then pressurized up to 17 bar. According to Henry's law and the ideal gas law, the reaction was run with the presence of $6.4 \mathrm{~g}$ ethylene in toluene and $9.7 \mathrm{~g}$ in the free space in the reactor. ${ }^{16}$ The reaction ran for $3 \mathrm{~h}$ at $25^{\circ} \mathrm{C}$, then quenched by venting the autoclave. To the solution, was added methanol- $\mathrm{HCl}$ in $4: 1 \mathrm{v} / \mathrm{v}$ ratio. Toluene was used to extract the organics and methanol or acetone was used to precipitate the 
Table 4 Selected bond distances $(\AA)$ and angles $\left({ }^{\circ}\right)$

\begin{tabular}{|c|c|c|c|c|c|c|c|}
\hline \multicolumn{8}{|c|}{$\left[\mathrm{Me}_{2} \mathrm{NCMe}_{2} \mathrm{CH}=\mathrm{N}\left(2,6-\mathrm{Me}_{2} \mathrm{C}_{6} \mathrm{H}_{3}\right)\right] \mathrm{NiBr}_{2}(\mathrm{Ni}-\mathbf{1 b})$} \\
\hline $\mathrm{Ni}-\mathrm{N} 1$ & $2.001(8)$ & $\mathrm{Ni}-\mathrm{N} 2$ & $2.050(8)$ & $\mathrm{Ni}-\mathrm{Br} 1$ & $2.3408(18)$ & $\mathrm{Ni}-\mathrm{Br} 2$ & $2.3405(18)$ \\
\hline $\mathrm{N} 1-\mathrm{C} 1$ & $1.250(12)$ & N2-C2 & $1.5000(13)$ & $\mathrm{C} 1-\mathrm{C} 2$ & $1.503(13)$ & N1-C21 & $1.455(12)$ \\
\hline $\mathrm{N} 2-\mathrm{C} 5$ & $1.507(13)$ & N2-C6 & $1.484(13)$ & & & & \\
\hline $\mathrm{N} 1-\mathrm{Ni}-\mathrm{N} 2$ & $81.9(3)$ & $\mathrm{Br} 1-\mathrm{Ni}-\mathrm{Br} 2$ & $116.80(7)$ & $\mathrm{Ni}-\mathrm{N} 1-\mathrm{C} 1$ & $112.5(7)$ & & \\
\hline $\mathrm{Ni}-\mathrm{N} 2-\mathrm{C} 2$ & 107.8(6) & N1-C1-C2 & $122.7(9)$ & N2-C2-C1 & $105.6(8)$ & & \\
\hline $\mathrm{C} 5-\mathrm{N} 2-\mathrm{Ni}$ & $113.6(6)$ & $\mathrm{C} 6-\mathrm{N} 2-\mathrm{Ni}$ & $106.1(6)$ & $\mathrm{C} 21-\mathrm{N} 1-\mathrm{Ni}$ & $125.3(6)$ & & \\
\hline \multicolumn{8}{|c|}{$\left[\mathrm{Me}_{2} \mathrm{NCMe}_{2} \mathrm{CH}=\mathrm{N}\left(2,6{ }^{i} \mathrm{Pr}_{2} \mathrm{C}_{6} \mathrm{H}_{3}\right)\right] \mathrm{NiBr}_{2}(\mathrm{Ni}-1 \mathrm{c})$} \\
\hline $\mathrm{Ni}-\mathrm{N} 1$ & $2.011(5)$ & $\mathrm{Ni}-\mathrm{N} 2$ & $2.061(6)$ & $\mathrm{Ni}-\mathrm{Br} 1$ & $2.3290(13)$ & $\mathrm{Ni}-\mathrm{Br} 2$ & $2.3521(17)$ \\
\hline $\mathrm{N} 1-\mathrm{C} 1$ & $1.267(8)$ & $\mathrm{N} 2-\mathrm{C} 2$ & $1.463(11)$ & $\mathrm{C} 1-\mathrm{C} 2$ & $1.492(10)$ & $\mathrm{N} 1-\mathrm{C} 21$ & $1.439(8)$ \\
\hline $\mathrm{N} 2-\mathrm{C} 5$ & $1.507(11)$ & $\mathrm{N} 2-\mathrm{C} 6$ & $1.550(13)$ & & & & \\
\hline $\mathrm{N} 1-\mathrm{Ni}-\mathrm{N} 2$ & $81.7(2)$ & $\mathrm{Br} 1-\mathrm{Ni}-\mathrm{Br} 2$ & $118.20(6)$ & $\mathrm{Ni}-\mathrm{N} 1-\mathrm{C} 1$ & $111.5(4)$ & & \\
\hline $\mathrm{Ni}-\mathrm{N} 2-\mathrm{C} 2$ & $108.3(5)$ & $\mathrm{N} 1-\mathrm{C} 1-\mathrm{C} 2$ & $122.8(6)$ & $\mathrm{N} 2-\mathrm{C} 2-\mathrm{C} 1$ & $106.6(5)$ & & \\
\hline $\mathrm{C} 5-\mathrm{N} 2-\mathrm{Ni}$ & $112.5(6)$ & $\mathrm{C} 6-\mathrm{N} 2-\mathrm{Ni}$ & $105.9(5)$ & $\mathrm{C} 21-\mathrm{N} 1-\mathrm{Ni}$ & $128.1(3)$ & & \\
\hline \multicolumn{8}{|c|}{$\left[\mathrm{Et}_{2} \mathrm{NCMe}_{2} \mathrm{CH}=\mathrm{NPh}\right] \mathrm{NiBr}_{2}(\mathrm{Ni}-\mathbf{2 a})$} \\
\hline $\mathrm{Ni}-\mathrm{N} 1$ & $1.997(4)$ & $\mathrm{Ni}-\mathrm{N} 2$ & $2.058(4)$ & $\mathrm{Ni}-\mathrm{Br} 1$ & $2.3535(7)$ & $\mathrm{Ni}-\mathrm{Br} 2$ & $2.3772(7)$ \\
\hline $\mathrm{N} 1-\mathrm{C} 1$ & $1.263(6)$ & $\mathrm{N} 2-\mathrm{C} 2$ & $1.530(5)$ & $\mathrm{C} 1-\mathrm{C} 2$ & $1.508(7)$ & $\mathrm{N} 1-\mathrm{C} 21$ & $1.434(6)$ \\
\hline $\mathrm{N} 2-\mathrm{C} 5$ & $1.505(5)$ & N2-C6 & $1.500(6)$ & & & & \\
\hline $\mathrm{N} 1-\mathrm{Ni}-\mathrm{N} 2$ & $82.41(15)$ & $\mathrm{Br} 1-\mathrm{Ni}-\mathrm{Br} 2$ & $123.61(3)$ & $\mathrm{Ni}-\mathrm{N} 1-\mathrm{C} 1$ & $114.5(3)$ & & \\
\hline $\mathrm{Ni}-\mathrm{N} 2-\mathrm{C} 2$ & $106.0(3)$ & $\mathrm{N} 1-\mathrm{C} 1-\mathrm{C} 2$ & $119.5(4)$ & $\mathrm{N} 2-\mathrm{C} 2-\mathrm{C} 1$ & $106.7(3)$ & & \\
\hline $\mathrm{C} 5-\mathrm{N} 2-\mathrm{Ni}$ & 108.1(3) & $\mathrm{C} 6-\mathrm{N} 2-\mathrm{Ni}$ & $111.0(3)$ & $\mathrm{C} 21-\mathrm{N} 1-\mathrm{Ni}$ & $123.7(3)$ & & \\
\hline \multicolumn{8}{|c|}{$\left[\mathrm{Et}_{2} \mathrm{NCMe}_{2} \mathrm{CH}=\mathrm{N}\left(2,6-\mathrm{Me}_{2} \mathrm{C}_{6} \mathrm{H}_{3}\right)\right] \mathrm{NiBr}_{2}(\mathrm{Ni}-\mathbf{2 b})$} \\
\hline $\mathrm{Ni}-\mathrm{N} 1$ & $1.991(3)$ & $\mathrm{Ni}-\mathrm{N} 2$ & $2.080(3)$ & $\mathrm{Ni}-\mathrm{Br} 1$ & $2.3666(6)$ & $\mathrm{Ni}-\mathrm{Br} 2$ & $2.3514(6)$ \\
\hline $\mathrm{N} 1-\mathrm{C} 1$ & $1.270(4)$ & $\mathrm{N} 2-\mathrm{C} 2$ & $1.537(5)$ & $\mathrm{C} 1-\mathrm{C} 2$ & $1.516(5)$ & $\mathrm{N} 1-\mathrm{C} 21$ & $1.449(4)$ \\
\hline $\mathrm{N} 2-\mathrm{C} 5$ & $1.504(5)$ & N2-C6 & $1.499(4)$ & & & & \\
\hline $\mathrm{N} 1-\mathrm{Ni}-\mathrm{N} 2$ & $82.66(11)$ & $\mathrm{Br} 1-\mathrm{Ni}-\mathrm{Br} 2$ & $118.33(2)$ & $\mathrm{Ni}-\mathrm{N} 1-\mathrm{Cl}$ & $112.3(2)$ & & \\
\hline $\mathrm{Ni}-\mathrm{N} 2-\mathrm{C} 2$ & $103.34(19)$ & $\mathrm{N} 1-\mathrm{C} 1-\mathrm{C} 2$ & $120.9(3)$ & $\mathrm{N} 2-\mathrm{C} 2-\mathrm{C} 1$ & $105.8(3)$ & & \\
\hline $\mathrm{C} 5-\mathrm{N} 2-\mathrm{Ni}$ & $106.7(2)$ & $\mathrm{C} 6-\mathrm{N} 2-\mathrm{Ni}$ & $109.5(2)$ & $\mathrm{C} 21-\mathrm{N} 1-\mathrm{Ni}$ & $127.7(2)$ & & \\
\hline \multicolumn{8}{|c|}{$\left[\left(c-\mathrm{C}_{3} \mathrm{H}_{6}\right) \mathrm{NCMe}_{2} \mathrm{C}=\mathrm{N}\left(2,6{ }^{i} \mathrm{Pr}_{2} \mathrm{C}_{6} \mathrm{H}_{3}\right)\right] \mathrm{NiBr}_{2}(\mathrm{Ni}-\mathbf{4 c})$} \\
\hline $\mathrm{Ni}-\mathrm{N} 1$ & $2.002(3)$ & $\mathrm{Ni}-\mathrm{N} 2$ & $2.049(4)$ & $\mathrm{Ni}-\mathrm{Br} 1$ & $2.3591(10)$ & $\mathrm{Ni}-\mathrm{Br} 2$ & $2.3307(10)$ \\
\hline $\mathrm{N} 1-\mathrm{C} 1$ & $1.276(6)$ & $\mathrm{N} 2-\mathrm{C} 2$ & $1.501(7)$ & $\mathrm{C} 1-\mathrm{C} 2$ & $1.494(7)$ & $\mathrm{N} 1-\mathrm{C} 21$ & $1.443(6)$ \\
\hline $\mathrm{N} 2-\mathrm{C} 5$ & $1.517(7)$ & $\mathrm{N} 2-\mathrm{C} 7$ & $1.504(8)$ & & & & \\
\hline $\mathrm{N} 1-\mathrm{Ni}-\mathrm{N} 2$ & $82.20(16)$ & $\mathrm{Br} 1-\mathrm{Ni}-\mathrm{Br} 2$ & 116.14(4) & $\mathrm{Ni}-\mathrm{N} 1-\mathrm{Cl}$ & $112.6(3)$ & & \\
\hline $\mathrm{Ni}-\mathrm{N} 2-\mathrm{C} 2$ & $107.8(3)$ & $\mathrm{N} 1-\mathrm{C} 1-\mathrm{C} 2$ & $121.6(4)$ & $\mathrm{N} 2-\mathrm{C} 2-\mathrm{C} 1$ & $106.6(4)$ & & \\
\hline $\mathrm{C} 5-\mathrm{N} 2-\mathrm{Ni}$ & $115.4(3)$ & $\mathrm{C} 7-\mathrm{N} 2-\mathrm{Ni}$ & $108.2(4)$ & $\mathrm{C} 21-\mathrm{N} 1-\mathrm{Ni}$ & $128.2(3)$ & & \\
\hline \multicolumn{8}{|c|}{$\left[\left(c-\mathrm{C}_{4} \mathrm{H}_{8}\right) \mathrm{NCMe}_{2} \mathrm{C}=\mathrm{N}\left(2,6-{ }^{i} \mathrm{Pr}_{2} \mathrm{C}_{6} \mathrm{H}_{3}\right)\right] \mathrm{NiBr}_{2}(\mathrm{Ni}-\mathbf{5} \mathrm{c})$} \\
\hline $\mathrm{Ni}-\mathrm{N} 1$ & $1.996(2)$ & $\mathrm{Ni}-\mathrm{N} 2$ & $2.076(3)$ & $\mathrm{Ni}-\mathrm{Br} 1$ & $2.3368(6)$ & $\mathrm{Ni}-\mathrm{Br} 2$ & $2.3307(6)$ \\
\hline $\mathrm{N} 1-\mathrm{C} 1$ & $1.264(4)$ & $\mathrm{N} 2-\mathrm{C} 2$ & $1.513(4)$ & $\mathrm{C} 1-\mathrm{C} 2$ & $1.504(5)$ & $\mathrm{N} 1-\mathrm{C} 21$ & $1.441(4)$ \\
\hline $\mathrm{N} 2-\mathrm{C} 5$ & $1.500(5)$ & N2-C6 & $1.501(4)$ & & & & \\
\hline $\mathrm{N} 1-\mathrm{Ni}-\mathrm{N} 2$ & $83.30(11)$ & $\mathrm{Br} 1-\mathrm{Ni}-\mathrm{Br} 2$ & $117.02(2)$ & $\mathrm{Ni}-\mathrm{N} 1-\mathrm{C} 1$ & $112.5(2)$ & & \\
\hline $\mathrm{Ni}-\mathrm{N} 2-\mathrm{C} 2$ & $106.2(2)$ & $\mathrm{N} 1-\mathrm{C} 1-\mathrm{C} 2$ & $122.3(3)$ & $\mathrm{N} 2-\mathrm{C} 2-\mathrm{C} 1$ & $107.4(3)$ & & \\
\hline $\mathrm{C} 5-\mathrm{N} 2-\mathrm{Ni}$ & $104.4(2)$ & $\mathrm{C} 6-\mathrm{N} 2-\mathrm{Ni}$ & $115.6(2)$ & $\mathrm{C} 21-\mathrm{N} 1-\mathrm{Ni}$ & $128.6(2)$ & & \\
\hline
\end{tabular}

PE. The GPC analysis was done to the soluble part in toluene solutions, relative to polystyrene standards.

\section{X-Ray crystallographic analysis}

The diffraction data were measured on a Nonius CAD-4, SmartCCD or Nonius KappaCCD diffractometer with graphitemonochromatized $\operatorname{MoK} \alpha$ radiation $(\lambda=0.7103 \AA)$. No significant decay was observed during the data collection. The structures were solved using the direct method and refined by full-matrix leastsquares on the $F^{2}$ value.
All the non-hydrogen atoms were refined anisotropically. Hydrogen atoms were identified by calculation and refined using a riding mode, and their contributions to structure factors were included. Atomic scattering factors were taken from the International Tables of Crystallographic Data, vol. IV. ${ }^{17}$ Computing programs are from the NRC VAX package. Crystallographic data and selected atomic coordinates and bond parameters are collected in Tables 3 and 4. One molecule of the asymmetric unit of Ni1b shows disorder in the amino moiety that has been refined with restrains. The prime labelled atoms account for $50 \%$ of occupancies. The rest of data are supplied in the supplementary material. $\dagger$ 


\section{Computational details}

All geometries were accomplished with QM optimization by means of the gradient techniques of Becke's three parameter hybrid functional incorporating the Lee-Yang-Parr correlation functional (BLYP) with VWN (Vosko, Wilk, and Nusair parameterization) local density approximation implemented in ADF(ADF 2000.02 and ADF 2004.01). The electronic configurations of the molecular systems were treated by a triple- $\xi$ STOs basis set with the $2 \mathrm{p}$ frozen core on Nickel; double- $\xi$ STOs basis set with the $1 \mathrm{~s}$ frozen core on nitrogen and carbon with a $3 \mathrm{~d}$ single polarization function, and double- $\xi$ STOs basis set on hydrogen with a $2 p$ polarization function. The results of both $(\mathbf{L}-\mathbf{1 b}) \mathrm{Ni}\left(\mathrm{C}_{2} \mathrm{H}_{4}\right)\left({ }^{n} \mathrm{Pr}\right)$ and $(\mathbf{L}-2 \mathbf{b}) \mathrm{Ni}\left(\mathrm{C}_{2} \mathrm{H}_{4}\right)\left({ }^{n} \mathrm{Pr}\right)$ show that the trans forms are 2.17 and $21.07 \mathrm{~kJ} \mathrm{~mol}^{-1}$ more stable than the cis forms, respectively.

\section{Acknowledgements}

We thank the National Science Council, Taiwan ROC, NWO/NSC project for the financial support and thank Nan-Ya Plastic Co. for the generous supply of MAO.

\section{References}

1 (a) G. J. P. Britovsek, V. C. Gibson and D. F. Wass, Angew. Chem., Int. Ed., 1999, 38, 428; (b) S. D. Ittel, L. K. Johnson and M. Brookhart, Chem. Rev., 2000, 100, 1169; (c) G. J. Domski, J. M. Rose, G. W. Coates, A. D. Bolig and M. Brookhart, Prog. Polym. Sci., 2007, 32, 30.

2 (a) H. G. Alt and A. Köppl, Chem. Rev., 2000, 100, 1205; (b) V. C. Gibson and S. K. Spitzmesser, Chem. Rev., 2003, 103, 283.

3 (a) L. K. Johnson, C. M. Killian and M. Brookhart, J. Am. Chem. Soc., 1995, 117, 6414; (b) S. Mecking, L. K. Johnson, L. Wang and M. Brookhart, J. Am. Chem. Soc., 1998, 120, 888; (c) D. P. Gates, S. K. Svejda, E. Onate, C. M. Killian, L. K. Johnson, P. S. White and M. Brookhart, Macromolecules, 2000, 33, 2320; (d) M. Schmid, R. Eberhardt, M. Klinga, M. Leskelä and B. Rieger, Organometallics, 2001, 20, 2321; (e) M. D. Leatherman, S. A. Svejda, L. K. Johnson and M. Brookhart, J. Am. Chem. Soc., 2003, 125, 3068; (f) D. H. Camacho, E. V. Salo, J. W. Ziller and Z. Guan, Angew. Chem., Int. Ed., 2004, 43, 1821; $(g)$ H.-R. Liu, P. T. Gomes, S. I. Costa, M. T. Duarte, R. Branquinho, A. C. Fernandes, J. C. W. Chien, R. P. Singh and M. M. Marques, J. Organomet. Chem., 2005, 690, 1314; $(h)$ A. E. Cherian, J. M. Rose, E. B. Lobkovsky and G. W. Coates, J. Am. Chem. Soc., 2005, 127, 13770; (i) D. H. Camacho and Z. Guan, Macromolecules, 2005, 38, 2544; (j) D. Meinhard, M. Wegner, G. Kipiani, A. Hearley, P. Reuter, S. Fischer, O. Marti and B. Rieger, J. Am. Chem. Soc., 2007, 129, 9182 .

4 (a) W. Keim, Angew. Chem., Int. Ed. Engl., 1990, 29, 235; (b) J. Heinicke, M. Z. He, A. Dal, H. F. Klein, O. Hetche, W. Keim, U. Flörke and H. J. Haupt, Eur. J. Inorg. Chem., 2000, 431; (c) V. C. Gibson, A. Tomov, A. J. P. White and D. J. Williams, Chem. Commun., 2001, 719; (d) J. M. Malinoski and M. Brookhart, Organometallics, 2003, 22, 5324; (e) J. M. Malinoski, P. S. White and M. Brookhart, Organometallics, 2003, 22 , 621; $(f)$ P. Kuhn, D. Semeril, C. Jeunesse, D. Matt, M. Neuburger and A. Mota, Chem.-Eur. J., 2006, 12, 5210; $(g)$ C. M. Reisinger, R. J. Nowack, D. Volkmer and B. Rieger, Dalton Trans., 2007, 272; (h) P. Kuhn, D. Semeril, D. Matt, M. J. Chetcuti and P. Lutz, Dalton Trans., 2007, 515.

5 (a) E. K. van den Beuken, W. J. J. Smeets, A. L. Spek and B. L. Feringa, Chem. Commun., 1998, 223; (b) J. C. Jenkins and M. Brookhart, J. Am. Chem. Soc., 2004, 126, 5827; (c) W. Ponikwar, S. Mihan, K. Sunkel and W. Beck, Z. Anorg. Allg. Chem., 2006, 632, 2299; (d) P. Kuhn, D. Semeril, C. Jeunesse, D. Matt, P. J. Lutz, R. Louis and M. Neuburger, Dalton Trans., 2006, 3647; (e) R. M. Ceder, G. Muller, M. Ordinas and J. I. Ordinas, Dalton Trans., 2007, 83; (f) I. Goettker-Schnetmann, P. Wehrmann, C. Roehr and S. Mecking, Organometallics, 2007, 26, 2348.

6 (a) P. Braunstein, M. D. Fryzuk, M. Le Dall, F. Naud, S. J. Rettig and F. Speiser, J. Chem. Soc., Dalton Trans., 2000, 1067; (b) K. R.
Reddy, K. Surekha, G.-H. Lee, S.-M. Peng, J.-T. Chen and S.-T. Liu, Organometallics, 2001, 20, 1292; (c) M. Sauthier, F. Leca, L. Toupet and R. Réau, Organometallics, 2002, 21, 1591; (d) C. Popeney and Z. Guan, Organometallics, 2005, 24, 1145; (e) M. Okada, Y. Nakayama, T. Ikeda and T. Shiono, Macromol. Rapid Commun., 2006, 27, 1418.

7 (a) T. R. Younkin, E. F. Connor, J. I. Henderson, S. K. Friedrich, R. H. Grubbs and D. A. Bansleben, Science, 2000, 287, 460; (b) F. M. Bauers and S. Mecking, Angew. Chem., Int. Ed., 2001, 40, 3020; (c) F. M. Bauers and S. Mecking, Macromolecules, 2001, 34, 1165; (d) F. A. Hicks and M. Brookhart, Organometallics, 2001, 20, 3217-3219; (e) D. Zhang, G.-X. Jin and N. Hu, Chem. Commun., 2002, 574; (f) Q.-Z. Yang, A. Kermagoret, M. Agostinho, O. Siri and P. Braunstein, Organometallics, 2006, 25, 5518; $(\mathrm{g})$ L. Zhang, M. Brookhart and P. S. White, Organometallics, 2006, 25, 1868.

8 (a) Z. Guan and W. J. Marshall, Organometallics, 2002, 21, 3580; (b) O. Daugulis and M. Brookhart, Organometallics, 2002, 21, 5926; (c) O. Daugulis, M. Brookhart and P. S. White, Organometallics, 2002, 21, 5935; (d) H.-P. Chen, Y.-H. Liu, S.-M. Peng and S.-T. Liu, Organometallics, 2003, 22, 4893; (e) F. Speiser, P. Braunstein, L. Saussine and R. Welter, Organometallics, 2004, 23, 2613; (f) F. Speiser, P. Braunstein and L. Saussine, Organometallics, 2004, 23, 2625; $(g)$ F. Speiser, P. Braunstein and L. Saussine, Organometallics, 2004, 23, 2633; (h) X. Tang, D. Zhang, S. Jie, W.-H. Sun and J. Chen, J. of Organomet. Chem., 2005, 690, 3918; (i) F. Speiser, P. Braunstein and L. Saussine, Acc. Chem. Res., 2005, 38, 784; (j) M. D. Doherty, S. Trudeau, P. S. White, J. P. Morken and M. Brookhart, Organometallics, 2007, 26, 1261.

9 (a) S. P. Meneghetti, P. J. Lutz and J. Kress, Organometallics, 1999, 18, 2734; (b) T. V. Laine, U. Piironen, K. Lappalainen, M. Klinga, E. Aitola and M. Leskelä, J. Organomet. Chem., 2000, 606, 112; (c) A. Köppl and H. G. Alt, J. Mol. Catal., 2000, 154, 45; (d) G. L. Tian, H. W. Boone and B. M. Novak, Macromolecules, 2001, 34, 7656; (e) M. S. Mohlala, I. A. Guzei, J. Darkwa and S. F. Mapolie, J. Mol. Catal., 2005, 241, 93; (f) Y. Li, L. Wang, H. Gao, F. Zhu and Q. Wu, Appl. Organomet. Chem., 2006, 20, 436; (g) P. Hao, S. Zhang, W.-H. Sun, Q. Shi, S. Adewuyi, X. $\mathrm{Lu}$ and P. Li, Organometallics, 2007, 26, 2439; $(h)$ L. Wang, C. Zhang and Z.-X. Wang, Eur. J. of Inorg. Chem., 2007, 2477; (i) R. S. Rojas, G. B. Galland, G. Wu and G. C. Bazan, Organometallics, 2007, 26, 5339; (j) H. Gao, Z. Ke, L. Pei, K. Song and Q. Wu, Polymer, 2007, 48, 7249; $(k)$ H. Gao, L. Pei, Y. Li, J. Zhang and Q. Wu, J. Mol. Catal., 2008, 280, 81.

10 (a) J. A. Sweet, J. M. Cavallari, W. A. Price, J. W. Ziller and D. V. McGrath, Tetrahedron: Asymmetry, 1997, 8, 207; (b) B. Y. Lee, G. C. Bazan, J. Vela, Z. J. A. Komon and X. Bu, J. Am. Chem. Soc., 2001, 123, 5352 .

11 J.-J. Lee, F.-Z. Yang, Y.-F. Lin, Y.-C. Chang, K.-H. Yu, M.-C. Chang, G.-H. Lee, Y.-H. Liu, Y. Wang, S.-T. Liu and J.-T. Chen, Dalton Trans., 2008, 5945-5956.

12 L. E. Fisher and J. M. Muchowski, Organic Preparation and Procedures Int., 1990, 22, 399

13 G. M. Sheldrick, SHELXL-97, Program for refinement of crystal structures, University of Göttingen, Germany, 1997.

14 For Ni-N ${ }_{\text {imine }}$ : (a) D. A. Handly, P. B. Hitchcock and G. J. Leigh, Inorg. Chim. Acta, 2001, 314, 1; (b) M. Sauthier, F. Leca, R. F. de Souza, K. Bernardo-Gusmão, L. F. T. Queiroz, L. Toupet and R. Réau, New J. Chem., 2002, 26, 630; (c) H. S. Schrekker, V. Kotov, P. PreishuberPflugl, P. White and M. Brookhart, Macromolecules, 2006, 39, 6341; (d) N. Muresan, T. Weyhermüller and K. Wieghardt, Dalton Trans., 2007, 4390.

15 (a) A. Michalak and T. Ziegler, Top. Organomet. Chem., 2005, 12, 145; (b) Z. Freixa and P. W. N. M. van Leeuwen, Dalton Trans., 2003, 1890; (c) P. W. N. M. van Leeuwen, P. C. J. Kmer, J. N. H. Reek and P. Dierkes, Chem. Rev., 2000, 100, 2741; (d) P. Dierkes and P. W. N. M. van Leeuwen, J. Chem. Soc., Dalton Trans., 1999, 1519; (e) L. Deng, T. K. Woo, L. Cavallo, P. L. Margl and T. Ziegler, J. Am. Chem. Soc., 1997, 119, 6177.

16 (a) A. L. McKnight and R. M. Waymouth, Macromolecules, 1999, 32, 2816; (b) I. Tritto, C. Marestin, L. Boggioni, M. C. Sacchi, H.-H. Brintzinger and D. R. Ferro, Macromolecules, 2001, 34, 5770.

17 D. T. Cromer, J. T. Waber, International Tables for X-Ray Crystallography, vol. IV, The Kynoch Press, Birmingham, England, 1974. 